\title{
Analysis of an Adaptive Finite Element Method for Recovering the Robin Coefficient
}

\author{
Yifeng Xu[ Jun Zou ${ }^{2}$
}

\begin{abstract}
Based on a new a posteriori error estimator, an adaptive finite element method is proposed for recovering the Robin coefficient involved in a diffusion system from some boundary measurement. The a posteriori error estimator can not be derived for this ill-posed nonlinear inverse problem as it was done for the existing a posteriori error estimators for direct problems. Instead, we shall derive the a posteriori error estimator from our convergence analysis of the adaptive algorithm. We prove that the adaptive algorithm guarantees a convergent subsequence of discrete solutions in an energy norm to some exact triplet (the Robin coefficient, state and costate variables) determined by the optimality system of the least-squares formulation with Tikhonov regularization for the concerned inverse problem. Some numerical results are also reported to illustrate the performance of the algorithm.
\end{abstract}

Keywords: Robin inverse problem, a posteriori error estimates, adaptive finite element method.

MSC(2010): $65 \mathrm{~N} 12,65 \mathrm{~N} 21,65 \mathrm{~N} 30$

\section{Introduction}

This work is concerned with estimation of the Robin coefficient associated with a diffusion equation. This is a severely ill-posed nonlinear inverse problem. To describe the inverse problem, we let $\Omega \subset \mathbb{R}^{d}(d=2,3)$ be an open bounded polyhedral domain, with its boundary $\Gamma$ made up of two connected disjoint open subsets satisfying $\bar{\Gamma}_{a} \cup \bar{\Gamma}_{i}=\Gamma$, both of which are a union of some $(d-1)$ dimensional polyhedral domains. The governing system of our interest is the diffusion equation:

$$
\begin{gathered}
-\nabla \cdot(\alpha \nabla u)=f \quad \text { in } \Omega, \\
\alpha \frac{\partial u}{\partial n}=q \quad \text { on } \Gamma_{a} ; \quad \alpha \frac{\partial u}{\partial n}+\gamma u=0 \quad \text { on } \Gamma_{i},
\end{gathered}
$$

where $f \in L^{2}(\Omega)$, coefficient $\alpha$ is assumed to be piecewisely $W^{1, \infty}$ such that $0<\alpha_{1} \leq \alpha \leq \alpha_{2}$ a.e. in $\Omega$ for two positive constants $\alpha_{1}$ and $\alpha_{2}, \boldsymbol{n}$ is the unit outward normal on $\Gamma$. In addition, $q$ is a prescribed flux in $L^{2}\left(\Gamma_{a}\right)$, and $\gamma$ is the Robin coefficient belonging to the admissible set

$$
\mathscr{A}=\left\{\gamma \in L^{\infty}\left(\Gamma_{i}\right): c_{0} \leq \gamma \leq c_{1} \text { a.e. on } \Gamma_{i}\right\}
$$

with $c_{0}$ and $c_{1}$ being two given positive constants.

The Robin boundary condition in (1.2) is encountered in many industrial applications, such as convection between the conducting body and the ambient environment in heat transfer [46], damage in corrosion detection [29] [32] and the metal-to-silicon contact in semiconductor device [8] [18], where the Robin coefficient represents the material profile on part of the boundary. As collecting accurate values of this coefficient is very expensive and difficult experimentally [46, some non-destructive

\footnotetext{
${ }^{1}$ Department of Mathematics, Scientific Computing Key Laboratory of Shanghai Universities and E-Institute for Computational Science of Shanghai Universities, Shanghai Normal University, Shanghai 200234, China. The research of this author was in part supported by NSFC (11201307), MOE of China through Specialized Research Fund for the Doctoral Program of Higher Education (20123127120001), E-Institute of Shanghai Universities (E03004) and Innovation Program of Shanghai Municipal Education Commission (13YZ059). (yfxu@shnu.edu.cn)

${ }^{2}$ Department of Mathematics, The Chinese University of Hong Kong, Shatin, N.T., Hong Kong. The work of this author was substantially supported by Hong Kong RGC grants (Projects 404611 and 405513) and a Direct Grant for Research from Chinese University of Hong Kong. (zou@math.cuhk.edu.hk)
} 
methods involving boundary or internal measurements are used in engineering [14] 37, which leads to the inverse problem of our interest: determine the Robin coefficient $\gamma$ with partial measurement data $g$ of $u$ on the accessible boundary $\Gamma_{a}$. Both identifiability and stability of this inverse problem have been investigated intensively [11] [12] [13] [20] [29].

In terms of numerical methods, there have been various techniques applied to the least-squares formulation with (1.1)-(1.2) as a constraint. This is a commonly used approach to deal with an inverse problem when measurement errors are present. The existing studies include the boundary integral method [19] [35, and the finite element methods [30] [31. Despite these efforts, it is still challenging to numerically estimate the Robin coefficient accurately and efficiently in a PDE-constrained optimization problem, particularly in the presence of discontinuity of the unknown Robin coefficient and the non-smooth boundaries. To resolve this difficulty, we propose in this work an adaptive finite element method (AFEM) for the inverse problem, in a hope that AFEM can achieve a desired accuracy for the numerical reconstruction with minimum degrees of freedom.

In practical computations, AFEM generates a sequence of nested triangulations and discrete solutions by successive loops:

$$
\text { SOLVE } \rightarrow \text { ESTIMATE } \rightarrow \text { MARK } \rightarrow \text { REFINE }
$$

This procedure is mainly driven by the module ESTIMATE consisting of a posteriori error estimation, i.e., some computable quantities formed by the discrete solution, the local mesh size and the given data. This field has been explored extensively for finite element approximations of direct partial differential equations and the relevant theory is well understood for elliptic problems; see the monographs [1] [45] and the references therein. Over the past decade, there have also been great developments in the a posteriori error analysis for PDE-constrained optimal control problems; see [3] 27] 28] 34 [36. Compared with optimal control problems, inverse problems are quite different in nature due to their severe instability with respect to the noise in the data. Several efforts have already been made in the direction; see e.g. [2] [4] [5] [21] [33].

Another crucial issue regarding AFEM is its convergence, does the iteration (1.3) ensure the convergence of the resulting approximate solutions? This issue has been investigated intensively for second order linear boundary value problems (see [7] [9] [38] [41] [42]), and for some nonlinear equations (see [6] [16] 222 [24] 25]). On the contrary, little has been done for inverse problems in this direction. The only related work is the one in [26] for a PDE-constrained optimal control problem, which studied the asymptotic error reduction property of an adaptive finite element approximation for the distributed control problems. The adaptive algorithm in [26] requires one extra step for some oscillation terms in the module MARK and the interior node property in the module REFINE. To the best of our knowledge, our earlier work [47] seems to be the only one that studies the convergence of an AFEM for an inverse problem. In [47], we considered the numerical reconstruction of distributed fluxes by an adaptive finite element method of the form 1.3 with the error estimator from [33] involved in ESTIMATE and several practical marking strategies adopted in MARK. Then it was proved that as the loop (1.3) proceeds, the sequence of discrete solutions generated by the adaptive algorithm converges to the exact solution in some norm and the error estimator also goes to zero. Unlike [26], the AFEM in [47] does not require the extra step for some oscillation terms in the module MARK and the interior node property in the module REFINE.

In this paper, we shall make a first effort to analyze the convergence of an adaptive finite element method for a nonlinear inverse problem, i.e., estimation of the Robin coefficient associated with (1.1)-1.2 from partial measurements on the accessible boundary $\Gamma_{a}$. To be more precise, we will demonstrate that the sequence of discrete solutions produced by an adaptive algorithm of the form (1.3) has a subsequence converging in some appropriate norm to an exact solution to the Robin inverse problem. Though the analysis on the current nonlinear ill-posed inverse problem is much more technical and difficult than the ones for direct problems, we can still manage to establish an 
AFEM here that is of the same effective framework as the standard one for direct elliptic problems (cf. [9] [39]), that is, no oscillation terms is involved in the module MARK and no interior node property is enforced in the module REFINE, therefore it is most favorable to practical computation. A unique feature of our analysis here is its derivation of the a posteriori error estimator. The existing approaches for direct partial differential equations and PDE-constrained optimal control problems fail for the current variational formulation associated with the Robin inverse problem due to the intrinsic nature in its severe ill-posedness and strong nonlinearity. Instead, we shall derive the a posteriori error estimator for the state, the costate and the Robin coefficient in the process of the convergence analysis for the new adaptive algorithm (see section 5).

In analyzing nearly all the existing adaptive algorithms that are based on a posteriori error estimator, a general procedure consists of three steps. The first step is to derive an error estimator that provides an upper and lower bound of the error between the exact solution and the finite element solution; The second step is to formulate an adaptive algorithm of the form (1.3) based on the a posteriori error estimator; The last step is to establish the convergence of the adaptive algorithm. Unfortunately the existing approaches to establish error estimators for direct problems (see, e.g., [1] [45]) or for inverse problems (see, e.g., [21] [33] [47]) do not work for our current nonlinear inverse problem. On one hand, the least-square functional for the Robin inverse problem is non-convex so that we can not deal with it like [21] [33] [47] or [34] [36. On the other hand, the Galerkin orthogonality is an essential property in a posteriori error analysis for direct problems, but it fails now as the optimality conditions contain a variational inequality associated with the Robin coefficient.

Because of the reasons above, we plan to consider the convergence directly by showing some limiting solution given by a successive iteration of the process 1.3 is an exact solution and derive some computable quantities during the convergence analysis, and these quantities are used as the error estimator in an adaptive algorithm. Moreover, this estimator is sufficient to guarantee the convergence of the resulting adaptive algorithm. This is one of the major novelties of the work, and it seems to be the first time to establish a posteriori error estimates from the perspective of convergence analysis. And we think this approach works for other nonlinear inverse problems as well.

Even though some of our arguments in convergence analysis follow partial existing principles (cf. 22] 23] 24] [38] [41]), there are several new yet essential difficulties and technical differences due to the strong nonlinearity of the inverse problem.

- The problems in [22] [23] [24] 38] [41] are all in the form of variational equations. However, the formulation of the Robin inverse problem in this work is a nonlinear constrained optimization problem, whose optimal conditions couple the state, the costate and the Robin coefficient (control) in a saddle-point system, including a variational inequality.

- To prove strong convergence of a subsequence of discrete solutions by the adaptive process (1.3) to some minimizer to a limiting optimization problem we utilize some techniques from nonlinear optimization, while the result for linear boundary value problems may be established by the standard finite element convergence theory (see, e.g., [38] [41]).

- To assert the limiting triplet (state, costate and control) is also an exact solution, we prove that it also satisfies the saddle-point system for the corresponding continuous optimization problem as in 22] for an eigenvalue problem and [24] for a quasi-linear elliptic equation. But unlike the existing works, the optimality conditions in the current situation include a variational inequality, so we have to first establish the convergence of a subsequence of the error indicators deduced from the relevant discrete inequality residuals of the adaptive discrete triplets and then come to our conclusion by some density argument.

We shall establish our convergence results in two steps. First, the sequence of discrete triplets 
(the approximate state, costate and Robin coefficient) produced by the adaptive algorithm is proved to contain a subsequence that converges strongly to some limiting triplet. It this step, we need to handle a nonlinear optimization problem with the system (1.1)- 1.2 as a constraint; see section 4 . The second step is to prove that the limiting triplet satisfies the saddle-point system of the Robin inverse problem, which in turn leads to the desired result. Here we consider and study limiting behaviors of residuals with respect to the approximate state, costate and Robin coefficient; see section 5. It is noted that an a posteriori error estimator to drive an adaptive process (1.3) for the Robin inverse problem is obtained simultaneously.

The rest of this paper is organized as follows. In section 2, we give a description of the Robin inverse problem in a variational formulation and its finite element method. A standard adaptive algorithm based on an a posteriori error estimator is introduced in section 3 and it is proved to generate a sequence of discrete triplets strongly converging to some limiting triplet in section 4 . Section 5 is devoted to the main result and the derivation of the error estimator. Two numerical examples for the algorithm are presented in section 6 . Finally, some concluding remarks are provided in section 7.

Throughout the paper we adopt the standard notation for the Lebesgue space $L^{p}(G)$ and the Sobolev space $W^{m, p}(G)$ as well as $H^{m}(G)(p=2)$ for integer $m \geq 0$ on an open bounded domain $G \subset \mathbb{R}^{d}$. Related norms and semi-norms of $W^{m, p}(G), H^{m}(G)$ and the norm of $L^{p}(G)$ are denoted by $\|\cdot\|_{m, p, G},\|\cdot\|_{m, G},|\cdot|_{m, G}$ and $\|\cdot\|_{L^{p}(G)}$ respectively. We use $(\cdot, \cdot)_{G}$ to denote the $L^{2}$ scalar product on a domain $G \subset \bar{\Omega}$. The subscript is omitted when $G=\Omega$. Moreover, we shall use $C$, with or without subscript, for a generic constant independent of the mesh size and it may take a different value at each occurrence.

\section{Mathematical formulation}

The Robin inverse problem of our interest is severely ill-posed [30] [31]. For a stable estimation of the Robin coefficient, we shall reformulate it as the following constrained optimization problem with the Tikhonov regularization:

$$
\inf _{\gamma \in \mathscr{A}} \mathcal{J}(\gamma)=\frac{1}{2}\|u(\gamma)-g\|_{0, \Gamma_{a}}^{2}+\frac{\beta}{2}\|\gamma\|_{0, \Gamma_{i}}^{2},
$$

where $\beta>0$ is a regularization parameter, and $u:=u(\gamma) \in H^{1}(\Omega)$ solves the variational formulation of $(1.1)-(1.2)$ :

$$
(\alpha \boldsymbol{\nabla} u, \boldsymbol{\nabla} \phi)+(\gamma u, \phi)_{\Gamma_{i}}=(f, \phi)+(q, \phi)_{\Gamma_{a}} \quad \forall \phi \in H^{1}(\Omega) .
$$

There exists at least one minimizer to the problem (2.1)-(2.2) [31. We note that over $H^{1}(\Omega)$ the norm $\left(\|\nabla \cdot\|_{0}^{2}+\|\cdot\|_{0, \Gamma_{i}}^{2}\right)^{1 / 2}$ is equivalent to the usual $H^{1}$-norm due to the Poincaré inequality. For efficient evaluation of the gradient in numerical simulation of the optimal problem (2.1) and (2.2), we often introduce a costate $p(\gamma)$ satisfying an adjoint problem for $(1.1)-(1.2)$ with respect to $u(\gamma)-g$ :

$$
\begin{gathered}
-\nabla \cdot(\alpha \nabla p)=0 \quad \text { in } \quad \Omega \\
\alpha \frac{\partial p}{\partial n}=u(\gamma)-g \quad \text { on } \quad \Gamma_{a} ; \quad \alpha \frac{\partial p}{\partial n}+\gamma p=0 \quad \text { on } \quad \Gamma_{i} .
\end{gathered}
$$

With the help of this adjoint system, the Gâteaux derivative of $\mathcal{J}(\gamma)$ at $\gamma \in \mathscr{A}$ in the direction $\lambda \in L^{\infty}\left(\Gamma_{i}\right)$ can be represented by (cf. [31])

$$
\mathcal{J}^{\prime}(\gamma)[\lambda]=(\beta \gamma-u(\gamma) p(\gamma), \lambda)_{\Gamma_{i}}
$$


Now with the above preparations and the introduction of a costate $p^{*}\left(\gamma^{*}\right) \in H^{1}(\Omega)$, the minimizer $\left(\gamma^{*}, u^{*}\left(\gamma^{*}\right)\right)$ to the problem (2.1)-2.2) is characterized by the following optimality conditions:

$$
\begin{aligned}
& \left(\alpha \boldsymbol{\nabla} u^{*}, \boldsymbol{\nabla} \phi\right)+\left(\gamma^{*} u^{*}, \phi\right)_{\Gamma_{i}}=(f, \phi)+(q, \phi)_{\Gamma_{a}} \quad \forall \phi \in H^{1}(\Omega), \\
& \left(\alpha \boldsymbol{\nabla} p^{*}, \boldsymbol{\nabla} v\right)+\left(\gamma^{*} p^{*}, v\right)_{\Gamma_{i}}=\left(u^{*}-g, v\right)_{\Gamma_{a}} \quad \forall v \in H^{1}(\Omega), \\
& \left(\beta \gamma^{*}-u^{*}\left(\gamma^{*}\right) p^{*}\left(\gamma^{*}\right), \lambda-\gamma^{*}\right)_{\Gamma_{i}} \geq 0 \quad \forall \lambda \in \mathscr{A} .
\end{aligned}
$$

We note that the equation (2.3) is the constraint $(2.2)$ for $u^{*}$ and the equation $(2.4)$ is the variational formulation of the adjoint problem for (1.1)- (1.2) with respect to $u^{*}-g$. Using the Gâteaux derivative of $\mathcal{J}(\gamma)$ at $\gamma^{*}$, we find that the variational inequality (2.5) is a necessary condition for the minimizer $\gamma^{*}$ to the problem (2.1) over a convex set $\mathscr{A}$.

Next we introduce a finite element method to approximate the continuous optimization problem (2.1)-(2.2). Let $\mathcal{T}_{h}$ be a shape-regular conforming triangulation of $\bar{\Omega}$ into a set of disjoint closed simplices with diameter $h_{T}:=|T|^{1 / d}$ for each $T \in \mathcal{T}_{h}$ such that the coefficient $\alpha$ is $W^{1, \infty}$ in each element. Let $V_{h}$ be the usual $H^{1}$-conforming linear element space over $\mathcal{T}_{h}, V_{h, \Gamma_{i}}:=\left.V_{h}\right|_{\Gamma_{i}}$ be the restriction of $V_{h}$ on $\Gamma_{i}$ and $\mathscr{A}_{h}:=V_{h, \Gamma_{i}} \cap \mathscr{A}$ be the discrete admissible set. Then we approximate the problem 2.1)-2.2 by

$$
\min _{\gamma_{h} \in \mathscr{A}_{h}} \mathcal{J}\left(\gamma_{h}\right)=\frac{1}{2}\left\|u_{h}\left(\gamma_{h}\right)-g\right\|_{0, \Gamma_{a}}^{2}+\frac{\beta}{2}\left\|\gamma_{h}\right\|_{0, \Gamma_{i}}^{2},
$$

where $u_{h}:=u_{h}\left(\gamma_{h}\right) \in V_{h}$ solves the discrete problem

$$
\left(\alpha \nabla u_{h}, \nabla \phi_{h}\right)+\left(\gamma_{h} u_{h}, \phi_{h}\right)_{\Gamma_{i}}=\left(f, \phi_{h}\right)+\left(q, \phi_{h}\right)_{\Gamma_{a}} \quad \forall \phi_{h} \in V_{h} .
$$

As in the continuous case, there exists at least one minimizer to 2.6)-2.7] 31, and the minimizer $\gamma_{h}^{*} \in \mathscr{A}_{h}$, the discrete state and costate $u_{h}^{*} \in V_{h}$ and $p_{h}^{*} \in V_{h}$ satisfy the optimality conditions:

$$
\begin{aligned}
& \left(\alpha \nabla u_{h}^{*}, \nabla \phi_{h}\right)+\left(\gamma_{h}^{*} u_{h}^{*}, \phi_{h}\right)_{\Gamma_{i}}=\left(f, \phi_{h}\right)+\left(q, \phi_{h}\right)_{\Gamma_{a}} \quad \forall \phi_{h} \in V_{h}, \\
& \left(\alpha \nabla p_{h}^{*}, \nabla v_{h}\right)+\left(\gamma_{h}^{*} p_{h}^{*}, v_{h}\right)_{\Gamma_{i}}=\left(u_{h}^{*}-g, v_{h}\right)_{\Gamma_{a}} \quad \forall v_{h} \in V_{h}, \\
& \left(\beta \gamma_{h}^{*}-u_{h}^{*}\left(\gamma_{h}^{*}\right) p_{h}^{*}\left(\gamma_{h}^{*}\right), \lambda_{h}-\gamma_{h}^{*}\right)_{\Gamma_{i}} \geq 0 \quad \forall \lambda_{h} \in \mathscr{A}_{h} .
\end{aligned}
$$

We end this section with two uniformly stability estimates for finite element approximations $u_{h}$ and $\left(u_{h}^{*}, p_{h}^{*}\right)$, which are easily obtained from the problems (2.7), (2.8) and (2.9):

$$
\left\|u_{h}\right\|_{1}+\left\|u_{h}^{*}\right\|_{1} \leq C\left(\|f\|_{0}+\|q\|_{0, \Gamma_{a}}\right), \quad\left\|p_{h}^{*}\right\|_{1} \leq C\left(\|f\|_{0}+\|q\|_{0, \Gamma_{a}}+\|g\|_{0, \Gamma_{a}}\right) .
$$

\section{Adaptive algorithm}

In this section, we propose an adaptive finite element for the problem (2.3)-(2.5). For this purpose, some more notation and definitions are needed.

The collection of all faces (resp. all interior faces) in $\mathcal{T}_{h}$ is denoted by $\mathcal{F}_{h}$ (resp. $\mathcal{F}_{h}(\Omega)$ ) and its restriction on $\Gamma_{a}$ and $\Gamma_{i}$ by $\mathcal{F}_{h}\left(\Gamma_{a}\right)$ and $\mathcal{F}_{h}\left(\Gamma_{i}\right)$ respectively. The scalar $h_{F}:=|F|^{1 /(d-1)}$ stands for the diameter of $F \in \mathcal{F}_{h}$, which is associated with a fixed normal unit vector $\boldsymbol{n}_{F}$ in the interior of $\Omega$ and $\boldsymbol{n}_{F}=\boldsymbol{n}$ on the boundary $\Gamma$. We use $D_{T}\left(\operatorname{resp} . D_{F}\right)$ for the union of all elements in $\mathcal{T}_{h}$ with non-empty intersection with element $T \in \mathcal{T}_{h}$ (resp. $F \in \mathcal{F}_{h}$ ). Furthermore, for any $F \in \mathcal{F}_{h}(\Omega)$ (resp. $\left.F \in \mathcal{F}_{h}\left(\Gamma_{a}\right) \cup \mathcal{F}_{h}\left(\Gamma_{i}\right)\right)$ we denote by $\omega_{F}$ the union of two elements in $\mathcal{T}_{h}$ sharing the common face $F$ (resp. the element with $F$ as a face).

For any $\left(\phi_{h}, v_{h}, \lambda_{h}\right) \in V_{h} \times V_{h} \times \mathscr{A}_{h}$, we define two element residuals for each $T \in \mathcal{T}_{h}$ and three face residuals for each face $F \in \mathcal{F}_{h}$ by

$$
R_{T, 1}\left(\phi_{h}\right)=f+\nabla \cdot\left(\alpha \nabla \phi_{h}\right), \quad R_{T, 2}\left(v_{h}\right)=\nabla \cdot\left(\alpha \nabla v_{h}\right),
$$




$$
\begin{gathered}
J_{F, 1}\left(\phi_{h}, \lambda_{h}\right)= \begin{cases}{\left[\alpha \boldsymbol{\nabla} \phi_{h} \cdot \boldsymbol{n}_{F}\right]} & \text { for } F \in \mathcal{F}_{h}(\Omega), \\
-q+\alpha \boldsymbol{\nabla} \phi_{h} \cdot \boldsymbol{n}_{F} & \text { for } F \in \mathcal{F}_{h}\left(\Gamma_{a}\right), \\
\lambda_{h} \phi_{h}+\alpha \boldsymbol{\nabla} \phi_{h} \cdot \boldsymbol{n}_{F} & \text { for } F \in \mathcal{F}_{h}\left(\Gamma_{i}\right),\end{cases} \\
J_{F, 2}\left(v_{h}, \phi_{h}, \lambda_{h}\right)= \begin{cases}{\left[\alpha \boldsymbol{\nabla} v_{h} \cdot \boldsymbol{n}_{F}\right]} & \text { for } F \in \mathcal{F}_{h}(\Omega), \\
\alpha \boldsymbol{\nabla} v_{h} \cdot \boldsymbol{n}_{F}-\left(\phi_{h}-g\right) & \text { for } F \in \mathcal{F}_{h}\left(\Gamma_{a}\right), \\
\lambda_{h} v_{h}+\alpha \boldsymbol{\nabla} v_{h} \cdot \boldsymbol{n}_{F} & \text { for } F \in \mathcal{F}_{h}\left(\Gamma_{i}\right),\end{cases}
\end{gathered}
$$

and

$$
J_{F, 3}\left(\phi_{h}, v_{h}, \lambda_{h}\right)=\beta \lambda_{h}-\phi_{h} v_{h} \quad \text { for } F \in \mathcal{F}_{h}\left(\Gamma_{i}\right),
$$

where $\left[\alpha \boldsymbol{\nabla} \phi_{h} \cdot \boldsymbol{n}_{F}\right]$ and $\left[\alpha \boldsymbol{\nabla} v_{h} \cdot \boldsymbol{n}_{F}\right]$ are the jumps across $F \in \mathcal{F}_{h}(\Omega)$. Then for any $\mathcal{S}_{h} \subseteq \mathcal{F}_{h}$, we introduce the error estimator

$$
\begin{aligned}
& \eta_{h}^{2}\left(\phi_{h}, v_{h}, \lambda_{h}, f, q, g, \mathcal{S}_{h}\right)+\xi_{h}\left(\phi_{h}, v_{h}, \lambda_{h}, \mathcal{S}_{h}\right) \\
:= & \sum_{F \in \mathcal{S}_{h}} \eta_{F, h}^{2}\left(\phi_{h}, v_{h}, \lambda_{h}, f, q, g\right)+\sum_{F \in \mathcal{S}_{h} \cap \mathcal{F}_{h}\left(\Gamma_{i}\right)} \xi_{F, h}\left(\phi_{h}, v_{h}, \lambda_{h}\right) \\
:= & \sum_{F \in \mathcal{S}_{h}}\left(\eta_{F, h, 1}^{2}\left(\phi_{h}, \lambda_{h}, f, q\right)+\eta_{F, h, 2}^{2}\left(v_{h}, \phi_{h}, \lambda_{h}, g\right)\right)+\sum_{F \in \mathcal{S}_{h} \cap \mathcal{F}_{h}\left(\Gamma_{i}\right)} \xi_{F, h}\left(\phi_{h}, v_{h}, \lambda_{h}\right)
\end{aligned}
$$

with

$$
\begin{aligned}
\eta_{F, h, 1}^{2}\left(\phi_{h}, \lambda_{h}, f, q\right) & :=\sum_{T \in \omega_{F}} h_{T}^{2}\left\|R_{T, 1}\left(\phi_{h}\right)\right\|_{0, T}^{2}+h_{F}\left\|J_{F, 1}\left(\phi_{h}, \lambda_{h}\right)\right\|_{0, F}^{2} \\
\eta_{F, h, 2}^{2}\left(v_{h}, \phi_{h}, \lambda_{h}, g\right) & :=\sum_{T \in \omega_{F}} h_{T}^{2}\left\|R_{T, 2}\left(\phi_{h}\right)\right\|_{0, T}^{2}+h_{F}\left\|J_{F, 2}\left(v_{h}, \phi_{h}, \lambda_{h}\right)\right\|_{0, F}^{2}
\end{aligned}
$$

and

$$
\xi_{F, h}\left(\phi_{h}, v_{h}, \lambda_{h}\right):=h_{F}^{2}\left\|J_{F, 3}\left(\phi_{h}, v_{h}, \lambda_{h}\right)\right\|_{L^{1}(F)} .
$$

When $\mathcal{S}_{h}=\mathcal{F}_{h}, \mathcal{S}_{h}$ will be dropped in the parameter list of the error estimator above.

Generally speaking, the error estimator is supposed to admit an upper bound of the error $u^{*}-u_{h}^{*}$, $p^{*}-p_{h}^{*}$ and $\gamma^{*}-\gamma_{h}^{*}$ in some norms as in the case of direct boundary value problems [45]. But in the current situation, we are unable to achieve such a result directly due to the high nonlinearity of the Robin inverse problem. Nevertheless, we shall utilize the estimator as an error measure in an adaptive algorithm for the minimization problem $(2.1)-(2.2)$ or the saddle-point system $(2.3)-(2.5)$. As we shall see in section 5, this measure will ensure the convergence of the resulting adaptive algorithm.

Now we are in a position to state the adaptive algorithm based on the above error estimator. ¿From now on we will write every dependence on triangulations by the number $k$ of the mesh refinements, and always use, unless specified otherwise, the pair $\left(u_{k}^{*}, \gamma_{k}^{*}\right)$ for the minimizer of the problem (2.6)-(2.7) with $h$ replaced by $k$.

Algorithm 3.1. Given a conforming initial mesh $\mathcal{T}_{0}$. Set $k:=0$.

1. (SOLVE) Solve the discrete problem (2.6) -2.7) on $\mathcal{T}_{k}$ for $\left(u_{k}^{*}, \gamma_{k}^{*}\right) \in V_{k} \times \mathscr{A}_{k}$.

2. (ESTIMATE) Compute the error estimator $\eta_{k}\left(u_{k}^{*}, p_{k}^{*}, \gamma_{k}^{*}, f, q, g\right)+\xi_{k}\left(u_{k}^{*}, p_{k}^{*}, \gamma_{k}^{*}\right)$ as defined in (3.1).

3. (MARK) Mark a subset $\mathcal{S}_{k} \subset \mathcal{F}_{k}$ containing at least one face $\widetilde{F}$ in $\mathcal{F}_{k}$ with the largest error indicator, i.e.,

$$
\eta_{\widetilde{F}, k}\left(u_{k}^{*}, p_{k}^{*}, \gamma_{k}^{*}, f, q, g\right)+\xi_{\widetilde{F}, k}\left(u_{k}^{*}, p_{k}^{*}, \gamma_{k}^{*}\right)=\max _{F \in \mathcal{F}_{k}}\left(\eta_{F, k}\left(u_{k}^{*}, p_{k}^{*}, \gamma_{k}^{*}, f, q, g\right)+\xi_{F, k}\left(u_{k}^{*}, p_{k}^{*}, \gamma_{k}^{*}\right)\right)
$$


4. (REFINE) Refine each triangle $T$ with at least one edge in $\mathcal{S}_{k}$ by bisection to get $\mathcal{T}_{k+1}$.

5. Set $k:=k+1$ and go to Step 1 .

At the end of this section, we state a stability estimate of local error indicators, which will be used in convergence analysis in section 5 .

Lemma 3.1. Let $\left\{\left(u_{k}^{*}, p_{k}^{*}, \gamma_{k}^{*}\right)\right\}$ be the sequence of discrete solutions given by Algorithm 3.1. Then there holds for the error indicator $\eta_{F, k}$ defined in (3.1) that

$$
\begin{aligned}
& \eta_{F, k}^{2}\left(u_{k}^{*}, p_{k}^{*}, \gamma_{k}^{*}, f, q, g\right) \\
\leq & C\left(\left\|u_{k}^{*}\right\|_{1, \omega_{F}}^{2}+\left\|p_{k}^{*}\right\|_{1, \omega_{F}}^{2}+h_{F}^{2}\|f\|_{0, \omega_{F}}^{2}+h_{F}\|g\|_{0, F \cap \Gamma_{a}}^{2}+h_{F}\|q\|_{0, F \cap \Gamma_{a}}^{2}\right) \quad \forall F \in \mathcal{F}_{k} .
\end{aligned}
$$

Proof. By the inverse estimate, local quasi-uniformity of $\mathcal{T}_{k}$ and the constraints of $\alpha$ and $\gamma_{k}^{*}$, we get

$$
\begin{gathered}
\eta_{F, k, 1}^{2}\left(u_{k}^{*}, \gamma_{k}^{*}, q\right) \leq C\left(\max _{T \in \omega_{F}}\|\alpha\|_{1, \infty, T}^{2}\left\|u_{k}^{*}\right\|_{1, \omega_{F}}^{2}+h_{F}^{2}\|f\|_{0, \omega_{F}}^{2}+h_{F}\|q\|_{0, F \cap \Gamma_{a}}^{2}\right), \\
\eta_{F, k, 2}^{2}\left(p_{k}^{*}, u_{k}^{*}, \gamma_{k}^{*}, g\right) \leq C\left(\left\|u_{k}^{*}\right\|_{1, \omega_{F}}^{2}+\max _{T \in \omega_{F}}\|\alpha\|_{1, \infty, T}^{2}\left\|p_{k}^{*}\right\|_{1, \omega_{F}}^{2}+h_{F}\|g\|_{0, F \cap \Gamma_{a}}^{2}\right),
\end{gathered}
$$

which, along with the definition (3.1), leads to the estimate (3.3).

Remark 3.1. One may notice that only a general principle is given for possible marking strategies to be used in Step 3 of Algorithm 3.1. In fact, the condition (3.2) there can be easily fulfilled by some commonly used marking strategies, such as the maximum strategy, the equidistribution strategy and the modified equidistribution strategy. In addition, the bisection in the module REFINE divides a given tetrahedron/triangle into two subtetrahedra/subtriangles of the same size such that the shaperegularity of $\left\{\mathcal{T}_{k}\right\}$ holds uniformly [39] [44]. In other words, all constants in our subsequent estimates depend only on the initial mesh and the given data.

\section{Limiting behavior}

In this section, we study the limiting behavior of the sequence $\left\{\left(u_{k}^{*}, p_{k}^{*}, \gamma_{k}^{*}\right)\right\}$ generated by Algorithm 3.1. It is noted that all results in this section are independent of any specific marking strategy in the module MARK. We start with a limiting space and a limiting admissible set

$$
V_{\infty}:=\overline{\bigcup_{k \geq 0} V_{k}} \text { (in } H^{1} \text {-norm) and } \mathscr{A}_{\infty}:=\overline{\bigcup_{k \geq 0} \mathscr{A}_{k}}\left(\text { in } L^{2}\left(\Gamma_{i}\right)\right. \text {-norm), }
$$

where $\left\{V_{k}\right\}$ and $\left\{\mathscr{A}_{k}\right\}$ are both induced by Algorithm 3.1. It is worth pointing out that $V_{\infty}$ and $\mathcal{A}_{\infty}$ are generally different from $H^{1}(\Omega)$ and $\mathcal{A}$ respectively since the sequence of underlying meshes is produced by the non-uniform refinement. To be precise, it is easy to check that $V_{\infty}$ is a closed subspace of $H^{1}(\Omega)$. For $\mathscr{A}_{\infty}$, we have the following lemma.

Lemma 4.1. $\mathscr{A}_{\infty}$ is a closed convex subset of $\mathscr{A}$.

Proof. The strong closedness of $\mathscr{A}_{\infty}$ comes directly from its definition. Now for any $\lambda$ and $\mu$ in $\mathscr{A}_{\infty}$, there exist two sequences $\left\{\lambda_{k}\right\}$ and $\left\{\mu_{k}\right\} \subset \bigcup_{k \geq 0} \mathscr{A}_{k}$ such that $\lambda_{k} \rightarrow \lambda$ and $\mu_{k} \rightarrow \mu$ in $L^{2}\left(\Gamma_{i}\right)$. Therefore, noting the convexity of $\mathscr{A}_{k}$, we have $\left\{t \lambda_{k}+(1-t) \mu_{k}\right\} \subset \bigcup_{k \geq 0} \mathscr{A}_{k}$ and $t \lambda_{k}+(1-t) \mu_{k} \rightarrow$ $t \lambda+(1-t) \mu$ in $L^{2}\left(\Gamma_{i}\right)$ for any $t \in[0,1]$. As a result, the convexity follows. Furthermore, we have $\lambda_{k} \rightarrow \lambda$ a.e. on $\Gamma_{i}$ after (possibly) passing to a subsequence, which, together with the constraint $c_{0} \leq \lambda_{k} \leq c_{1}$ on $\Gamma_{i}$, implies that $c_{0} \leq \lambda \leq c_{1}$ a.e. on $\Gamma_{i}$. This proves that $\mathscr{A}_{\infty} \subset \mathscr{A}$. 
Now we introduce a counterpart of the minimization problem $2.6-2.7)$ over $\mathscr{A}_{\infty}$ :

$$
\inf _{\gamma \in \mathscr{A} \infty} \mathcal{J}(\gamma)=\frac{1}{2}\left\|u_{\infty}(\gamma)-g\right\|_{0, \Gamma_{a}}^{2}+\frac{\beta}{2}\|\gamma\|_{0, \Gamma_{i}}^{2}
$$

where $u_{\infty}:=u_{\infty}(\gamma) \in V_{\infty}$ satisfies the variational problem:

$$
\left(\alpha \nabla u_{\infty}, \nabla \phi\right)+\left(\gamma u_{\infty}, \phi\right)_{\Gamma_{i}}=(f, \phi)+(q, \phi)_{\Gamma_{a}} \quad \forall \phi \in V_{\infty}
$$

Theorem 4.1. There exists at least one minimizer to the optimization problem (4.1)-(4.2).

Proof. Let $\left\{\gamma^{n}\right\} \subset \mathscr{A}_{\infty}$ be a minimizing sequence satisfying $\mathcal{J}\left(\gamma^{n}\right) \rightarrow \inf \mathcal{J}(\gamma)$. As $\mathscr{A}_{\infty}$ is a closed convex set in $L^{2}\left(\Gamma_{i}\right)$, there exists a subsequence, still denoted by $\gamma^{n}$, and some $\gamma^{*} \in \mathscr{A}_{\infty}$ such that

$$
\gamma^{n} \rightarrow \gamma^{*} \quad \text { weakly in } L^{2}\left(\Gamma_{i}\right)
$$

With $\gamma=\gamma^{n}$ in 4.2$), u^{n}:=u^{n}\left(\gamma^{n}\right) \in V_{\infty}$ satisfies

$$
\left(\alpha \nabla u^{n}, \nabla \phi\right)+\left(\gamma^{n} u^{n}, \phi\right)_{\Gamma_{i}}=(f, \phi)+(q, \phi)_{\Gamma_{a}} \quad \forall \phi \in V_{\infty} .
$$

Taking $\phi=u^{n}$ in (4.4), we obtain by the norm equivalence and the trace theorem that $\left\|u^{n}\right\|_{1}$ is uniformly bounded independent of $n$. Therefore, there exists a subsequence, also denoted by $\left\{u^{n}\right\}$ and some $u^{*} \in V_{\infty}$ as $V_{\infty}$ is weakly closed such that

$$
u^{n} \rightarrow u^{*} \quad \text { weakly in } H^{1}(\Omega) ; \quad u^{n} \rightarrow u^{*} \quad \text { in } L^{2}(\Gamma) .
$$

Next we prove $u^{*}=u\left(\gamma^{*}\right)$. Letting $n$ go to infinity and noting the assumption on $\gamma^{n}$ and $u^{*}, \phi \in$ $L^{4}\left(\Gamma_{i}\right)$ due to the trace theorem, we deduce from the convergence results in 4.3 ) and $(4.5)$ :

$$
\begin{gathered}
\left(\alpha \nabla u^{n}, \nabla \phi\right) \rightarrow\left(\alpha \nabla u^{*}, \nabla \phi\right), \\
\left(\gamma^{n} u^{n}, \phi\right)_{\Gamma_{i}}=\left(\gamma^{n} u^{*}, \phi\right)_{\Gamma_{i}}+\left(\gamma^{n}\left(u^{n}-u^{*}\right), \phi\right)_{\Gamma_{i}} \rightarrow\left(\gamma^{*} u^{*}, \phi\right)_{\Gamma_{i}}
\end{gathered}
$$

which imply

$$
\left(\alpha \boldsymbol{\nabla} u^{*}, \boldsymbol{\nabla} \phi\right)+\left(\gamma^{*} u^{*}, \phi\right)_{\Gamma_{i}}=(f, \phi)+(q, \phi)_{\Gamma_{a}} \quad \forall \phi \in V_{\infty} .
$$

Finally, the standard argument, together with the strong convergence in 4.5) and the weak lower semi-continuity of $\|\cdot\|_{0, \Gamma_{i}}$, yields that $\gamma^{*}$ is a minimizer of the cost functional $\mathcal{J}(\cdot)$ over $\mathscr{A}_{\infty}$.

To present the main results of this section, we need some auxiliary results in the following two lemmas.

Lemma 4.2. Let $\left\{V_{k} \times \mathscr{A}_{k}\right\}$ be a sequence of discrete spaces and discrete sets generated by Algorithm 3.1. If the sequence $\left\{\gamma_{k}\right\} \subset \bigcup_{k \geq 0} \mathscr{A}_{k}$ converges weakly to some $\gamma^{*} \in \mathscr{A}_{\infty}$ in $L^{2}\left(\Gamma_{i}\right)$, then there exists a subsequence $\left\{\gamma_{k_{n}}\right\}$, such that for the sequence $\left\{u_{k_{n}}\left(\gamma_{k_{n}}\right)\right\} \subset \bigcup_{k \geq 0} V_{k}$ produced by (2.7) with $h$ replaced by $k_{n}$ and $u_{\infty}\left(\gamma^{*}\right) \in V_{\infty}$ generated by (4.2) with $\gamma=\gamma^{*}$ there holds that

$$
u_{k_{n}}\left(\gamma_{k_{n}}\right) \rightarrow u_{\infty}\left(\gamma^{*}\right) \text { in } L^{2}(\Gamma)
$$

If the sequence $\left\{\gamma_{k}\right\} \subset \bigcup_{k \geq 0} \mathscr{A}_{k}$ converges strongly to some $\gamma^{*} \in \mathscr{A}_{\infty}$ in $L^{2}\left(\Gamma_{i}\right)$, then for the whole sequence $\left\{u_{k}\left(\gamma_{k}\right)\right\} \subset \bigcup_{k \geq 0} V_{k}$ given by 2.7 with $h$ replaced by $k$ and $u_{\infty}\left(\gamma^{*}\right) \in V_{\infty}$ given by 4.2 with $\gamma=\gamma^{*}$ there holds

$$
u_{k}\left(\gamma_{k}\right) \rightarrow u_{\infty}\left(\gamma^{*}\right) \quad \text { in } H^{1}(\Omega)
$$


Proof. Taking $\phi_{k}=u_{k}\left(\gamma_{k}\right)$ in 2.7), we immediately know from 2.11 that $\left\|u_{k}\left(\gamma_{k}\right)\right\|_{1}$ is uniformly bounded independently of $k$, hence there exists a subsequence, denoted by $\left\{u_{k_{n}}\left(\gamma_{k_{n}}\right)\right\}$, and some $u^{*} \in H^{1}(\Omega)$ such that

$$
u_{k_{n}}\left(\gamma_{k_{n}}\right) \rightarrow u^{*} \quad \text { weakly in } H^{1}(\Omega) ; \quad u_{k_{n}}\left(\gamma_{k_{n}}\right) \rightarrow u^{*} \quad \text { in } L^{2}(\Gamma) .
$$

As $V_{\infty}$ is weakly closed, $u^{*} \in V_{\infty}$. For any integer $l \geq 0$, if we choose $k_{n} \geq l$ we know from (2.7) that

$$
\left(\alpha \nabla u_{k_{n}}\left(\gamma_{k_{n}}\right), \nabla \phi_{l}\right)+\left(\gamma_{k_{n}} u_{k_{n}}\left(\gamma_{k_{n}}\right), \phi_{l}\right)_{\Gamma_{i}}=\left(f, \phi_{l}\right)+\left(q, \phi_{l}\right)_{\Gamma_{i}} \quad \forall \phi_{l} \in V_{l} .
$$

Letting $n$ go to infinity and noting the convergence results in 4.8 as well as the weak convergence of $\left\{\gamma_{k}\right\}$, we find

$$
\begin{gathered}
\left(\alpha \nabla u_{k_{n}}\left(\gamma_{k_{n}}\right), \nabla \phi_{l}\right) \rightarrow\left(\alpha \nabla u^{*}, \boldsymbol{\nabla} \phi_{l}\right), \\
\left(\gamma_{k_{n}} u_{k_{n}}\left(\gamma_{k_{n}}\right), \phi_{l}\right)_{\Gamma_{i}}=\left(\gamma_{k_{n}} u^{*}, \phi_{l}\right)_{\Gamma_{i}}+\left(\gamma_{k_{n}}\left(u_{k_{n}}\left(\gamma_{k_{n}}\right)-u^{*}\right), \phi_{l}\right)_{\Gamma_{i}} \rightarrow\left(\gamma^{*} u^{*}, \phi_{l}\right)_{\Gamma_{i}},
\end{gathered}
$$

which imply

$$
\left(\alpha \nabla u^{*}, \nabla \phi_{l}\right)+\left(\gamma^{*} u^{*}, \phi_{l}\right)_{\Gamma_{i}}=\left(f, \phi_{l}\right)+\left(q, \phi_{l}\right)_{\Gamma_{i}} \quad \forall \phi_{l} \in V_{l} .
$$

As $l$ is arbitrary and $\phi_{l} \in V_{l}$, we easily see

$$
\left(\alpha \nabla u^{*}, \nabla \phi\right)+\left(\gamma^{*} u^{*}, \phi\right)_{\Gamma_{i}}=(f, \phi)+(q, \phi)_{\Gamma_{i}} \quad \forall \phi \in V_{\infty} .
$$

Now the first claim holds with $u^{*}=u_{\infty}\left(\gamma^{*}\right)$ in the second convergence result in (4.8).

To show the second convergence (4.7), we begin with an auxiliary discrete problem: Find $u_{k}\left(\gamma^{*}\right) \in$ $V_{k}$ such that

$$
\left(\alpha \boldsymbol{\nabla} u_{k}\left(\gamma^{*}\right), \boldsymbol{\nabla} \phi\right)+\left(\gamma^{*} u_{k}\left(\gamma^{*}\right), \phi\right)_{\Gamma_{i}}=(f, \phi)+(q, \phi)_{\Gamma_{a}} \quad \forall \phi \in V_{k} .
$$

Subtracting (2.7) from (4.9) with $\phi=u_{k}\left(\gamma^{*}\right)-u_{k}\left(\gamma_{k}\right)$ and using the generalized Hölder inequality, the Sobolev embedding theorem and the stability estimate (2.11), we come to

$$
\begin{aligned}
\left\|u_{k}\left(\gamma^{*}\right)-u_{k}\left(\gamma_{k}\right)\right\|_{1}^{2} & \leq C\left(\left(\gamma_{k}-\gamma^{*}\right) u_{k}\left(\gamma_{k}\right), u_{k}\left(\gamma^{*}\right)-u_{k}\left(\gamma_{k}\right)\right) \\
& \leq C\left\|\gamma^{*}-\gamma_{k}\right\|_{0, \Gamma_{i}}\left\|u_{k}\left(\gamma_{k}\right)\right\|_{L^{4}\left(\Gamma_{i}\right)}\left\|u_{k}\left(\gamma^{*}\right)-u_{k}\left(\gamma_{k}\right)\right\|_{L^{4}\left(\Gamma_{i}\right)} \\
& \leq C\left\|\gamma^{*}-\gamma_{k}\right\|_{0, \Gamma_{i}}\left\|u_{k}\left(\gamma^{*}\right)-u_{k}\left(\gamma_{k}\right)\right\|_{1} .
\end{aligned}
$$

i.e.,

$$
\left\|u_{k}\left(\gamma^{*}\right)-u_{k}\left(\gamma_{k}\right)\right\|_{1} \leq C\left\|\gamma^{*}-\gamma_{k}\right\|_{L^{2}\left(\Gamma_{i}\right)} .
$$

On the other hand, we note that 4.9$)$ is a finite element approximation of 4.2 with $\gamma=\gamma^{*} \in \mathscr{A}_{\infty}$, so the Cea's lemma admits an optimal approximation property

$$
\left\|u_{\infty}\left(\gamma^{*}\right)-u_{k}\left(\gamma^{*}\right)\right\|_{1} \leq C \inf _{v \in V_{k}}\left\|u_{\infty}\left(\gamma^{*}\right)-v\right\|_{1}
$$

Now the desired convergence (4.7) is a consequence of the above two estimates and the density of $\bigcup_{k \geq 0} V_{k}$ in $V_{\infty}$.

Next comes the first main result of this section.

Theorem 4.2. Let $\left\{V_{k} \times \mathscr{A}_{k}\right\}$ be a sequence of discrete spaces and discrete sets generated by Algorithm 3.1 and $\left\{\gamma_{k}^{*}\right\}$ be the corresponding sequence of minimizers to the discrete problem (2.6)-(2.7). Then the sequence $\left\{\gamma_{k}^{*}\right\}$ has a subsequence $\left\{\gamma_{k_{n}}^{*}\right\}$ converging strongly in $L^{2}\left(\Gamma_{i}\right)$ to a minimizer $\gamma_{\infty}^{*} \in \mathscr{A}_{\infty}$ of the problem (4.1)- 4.2 . 
Proof. Since $\left\{\gamma_{k}^{*}\right\}$ is uniformly bounded in $L^{2}\left(\Gamma_{i}\right)$ there exist a subsequence (still denoted by $\left\{\gamma_{k}^{*}\right\}$ ) and some $\gamma^{*} \in \mathscr{A}_{\infty}$ such that

$$
\gamma_{k}^{*} \rightarrow \gamma^{*} \quad \text { weakly in } L^{2}\left(\Gamma_{i}\right)
$$

Then we know from Lemma 4.2 by extracting a subsequence that

$$
u_{k_{n}}^{*}\left(\gamma_{k_{n}}^{*}\right) \rightarrow u_{\infty}\left(\gamma^{*}\right) \in V_{\infty} \quad \text { in } L^{2}\left(\Gamma_{a}\right)
$$

Furthermore, for any $\gamma \in \mathscr{A}_{\infty}$ there exists a sequence $\left\{\gamma_{l}\right\} \subset \bigcup_{k \geq 0} \mathscr{A}_{k}$ such that

$$
\lim _{l \rightarrow \infty}\left\|\gamma_{l}-\gamma\right\|_{0, \Gamma_{i}}=0
$$

which, along with 4.7 in Lemma 4.2 and the trace theorem, implies

$$
\lim _{l \rightarrow \infty}\left\|u_{l}\left(\gamma_{l}\right)-g\right\|_{0, \Gamma_{a}}^{2}=\left\|u_{\infty}(\gamma)-g\right\|_{0, \Gamma_{a}}^{2}
$$

Noting the whole sequence $\left\{\gamma_{k}^{*}\right\}$ are minimizers of $\mathcal{J}(\cdot)$ over $\left\{\mathscr{A}_{k}\right\}$, we know

$$
\mathcal{J}\left(\gamma_{k}^{*}\right) \leq \mathcal{J}\left(\gamma_{l}\right)=\frac{1}{2}\left\|u_{l}\left(\gamma_{l}\right)-g\right\|_{0, \Gamma_{a}}^{2}+\frac{\beta}{2}\left\|\gamma_{l}\right\|_{0, \Gamma_{i}}^{2}
$$

when $k \geq l$ for sufficiently large $l$. Then a collection of 4.10-4.13 gives

$$
\begin{aligned}
\mathcal{J}\left(\gamma^{*}\right) & =\frac{1}{2}\left\|u_{\infty}\left(\gamma^{*}\right)-g\right\|_{0, \Gamma_{a}}^{2}+\frac{\beta}{2}\left\|\gamma^{*}\right\|_{0, \Gamma_{i}}^{2} \\
& \leq \lim _{n \rightarrow \infty} \frac{1}{2}\left\|u_{k_{n}}\left(\gamma_{k_{n}}^{*}\right)-g\right\|_{0, \Gamma_{a}}^{2}+\liminf _{n \rightarrow \infty} \frac{\beta}{2}\left\|\gamma_{k_{n}}^{*}\right\|_{0, \Gamma_{i}}^{2} \\
& \leq \liminf _{n \rightarrow \infty} \mathcal{J}\left(\gamma_{k_{n}}^{*}\right) \leq \limsup _{n \rightarrow \infty} \mathcal{J}\left(\gamma_{k_{n}}^{*}\right) \leq \limsup _{k \rightarrow \infty} \mathcal{J}\left(\gamma_{k}^{*}\right) \leq \limsup _{l \rightarrow \infty} \mathcal{J}\left(\gamma_{l}\right)=\mathcal{J}(\gamma)
\end{aligned}
$$

for any $\gamma \in \mathscr{A}_{\infty}$. This indicates that $\gamma^{*}$ is a minimizer of the problem 4.1)-4.2). The choice $\gamma=\gamma^{*}$ in the above estimate yields equality $\lim _{n \rightarrow \infty} \mathcal{J}\left(\gamma_{k_{n}}^{*}\right)=\mathcal{J}\left(\gamma^{*}\right)=\inf \mathcal{J}\left(\mathscr{A}_{\infty}\right)$, which, along with 4.11), implies that $\lim _{n \rightarrow \infty}\left\|\gamma_{k_{n}}^{*}\right\|_{0, \Gamma_{i}}^{2}=\left\|\gamma_{\infty}^{*}\right\|_{0, \Gamma_{i}}^{2}$. Now the desired strong convergence follows from 4.10.

¿From the above theorem, it can be observed that if the sequence $\left\{V_{k} \times \mathscr{A}_{k}\right\}$ is given over uniformly refined triangulations, the strong convergence of $\gamma_{k}^{*}$ to $\gamma^{*}$ holds directly since we have naturally $H^{1}(\Omega)=\overline{\bigcup_{k \geq 0} V_{k}}$ in $H^{1}$-norm and $\mathscr{A}=\overline{\bigcup_{k \geq 0} \mathscr{A}_{k}}$ in $L^{2}\left(\Gamma_{i}\right)$-norm. But this is generally not true for a sequence of adaptively generated meshes. To achieve the desired convergence, we have to show that $\gamma_{\infty}^{*}$ is also a minimizer of the problem (2.1)-(2.2). Noting that Algorithm 3.1 involves the solution of the discrete saddle-point system (2.8)-(2.10), we introduce a costate $p_{\infty}^{*} \in V_{\infty}$ like the continuous case, then this costate, together with the minimizer $\gamma_{\infty}^{*} \in \mathscr{A}_{\infty}$ and the related state $u_{\infty}^{*} \in V_{\infty}$ of the problem 4.1)-4.2 solves the following system:

$$
\begin{aligned}
& \left(\alpha \nabla u_{\infty}^{*}, \nabla \phi\right)+\left(\gamma_{\infty}^{*} u_{\infty}^{*}, \phi\right)_{\Gamma_{i}}=(f, \phi)+(q, \phi)_{\Gamma_{a}} \quad \forall \phi \in V_{\infty} \\
& \left(\alpha \nabla p_{\infty}^{*}, \nabla v\right)+\left(\gamma_{\infty}^{*} p_{\infty}^{*}, v\right)_{\Gamma_{i}}=\left(u_{\infty}^{*}-g, v\right)_{\Gamma_{a}} \quad \forall v \in V_{\infty} \\
& \left(\beta \gamma_{\infty}^{*}-u_{\infty}^{*}\left(\gamma_{\infty}^{*}\right) p_{\infty}^{*}\left(\gamma_{\infty}^{*}\right), \lambda-\gamma_{\infty}^{*}\right)_{\Gamma_{i}} \geq 0 \quad \forall \lambda \in \mathscr{A}_{\infty} .
\end{aligned}
$$

It is easy to verify that the solution $\left(u_{\infty}^{*}, p_{\infty}^{*}\right)$ to 4.14 - 4.15 admits the stability estimates:

$$
\left\|u_{\infty}^{*}\right\|_{1} \leq C\left(\|f\|_{0}+\|q\|_{0, \Gamma_{a}}\right), \quad\left\|p_{\infty}^{*}\right\|_{1} \leq C\left(\|f\|_{0}+\|q\|_{0, \Gamma_{a}}+\|g\|_{0, \Gamma_{a}}\right) .
$$

Now we end this section with the second main result of this section for the above system. 
Theorem 4.3. Let $\left\{V_{k} \times \mathscr{A}_{k}\right\}$ be a sequence of discrete spaces and discrete sets generated by Algorithm 3.1, then the sequence $\left\{\left(u_{k}^{*}, p_{k}^{*}, \gamma_{k}^{*}\right)\right\}$ of discrete solutions to the system (2.8)-(2.10) has a subsequence $\left\{\left(u_{k_{n}}^{*}, p_{k_{n}}^{*}, \gamma_{k_{n}}^{*}\right)\right\}$ which converges to $\left(u_{\infty}^{*}, p_{\infty}^{*}, \gamma_{\infty}^{*}\right) \in V_{\infty} \times V_{\infty} \times \mathscr{A}_{\infty}$, a solution of the problem (4.14)4.16, in the following sense:

$$
\left\|u_{k_{n}}^{*}-u_{\infty}^{*}\right\|_{1} \rightarrow 0, \quad\left\|p_{k_{n}}^{*}-p_{\infty}^{*}\right\|_{1} \rightarrow 0, \quad\left\|\gamma_{k_{n}}^{*}-\gamma_{\infty}^{*}\right\|_{0, \Gamma_{i}} \rightarrow 0 \quad \text { as } n \rightarrow \infty
$$

Proof. The last convergence in (4.18) follows directly from Theorem 4.2, while the first one is a consequence of Lemma 4.2 (cf. (4.7)). To demonstrate the second convergence in 4.18), we introduce a solution $\widetilde{p}_{k_{n}} \in V_{k_{n}}$ to the following auxiliary problem:

$$
\left(\alpha \nabla \widetilde{p}_{k_{n}}, \nabla v\right)+\left(\gamma_{\infty}^{*} \widetilde{p}_{k_{n}}, v\right)_{\Gamma_{i}}=\left(u_{\infty}^{*}-g, v\right)_{\Gamma_{a}} \quad \forall v \in V_{k_{n}} .
$$

Combining (2.9) and 4.19) with $v=\widetilde{p}_{k_{n}}-p_{k_{n}}^{*}$ and arguing as in the proof of Lemma 4.2, we obtain

$$
\begin{aligned}
\left\|\widetilde{p}_{k_{n}}-p_{k_{n}}^{*}\right\|_{1}^{2} & \left.\leq C\left[\left(\gamma_{k_{n}}^{*}-\gamma_{\infty}^{*}\right) p_{k_{n}}^{*}, \widetilde{p}_{k_{n}}-p_{k_{n}}^{*}\right)_{\Gamma_{i}}+\left(u_{k_{n}}^{*}-u_{\infty}^{*}, \widetilde{p}_{k_{n}}-p_{k_{n}}^{*}\right)_{\Gamma_{a}}\right] \\
& \leq C\left(\left\|\gamma_{k_{n}}^{*}-\gamma_{\infty}^{*}\right\|_{0, \Gamma_{i}}\left\|p_{k_{n}}^{*}\right\|_{L^{4}\left(\Gamma_{i}\right)}\left\|\widetilde{p}_{k_{n}}-p_{k_{n}}^{*}\right\|_{L^{4}\left(\Gamma_{i}\right)}+\left\|u_{k_{n}}^{*}-u_{\infty}^{*}\right\|_{0, \Gamma_{a}}\left\|\widetilde{p}_{k_{n}}-p_{k_{n}}^{*}\right\|_{0, \Gamma_{a}}\right) \\
& \leq C\left(\left\|\gamma_{k_{n}}^{*}-\gamma_{\infty}^{*}\right\|_{0, \Gamma_{i}}+\left\|u_{k_{n}}^{*}-u_{\infty}^{*}\right\|_{0, \Gamma_{a}}\right)\left\|\widetilde{p}_{k_{n}}-p_{k_{n}}^{*}\right\|_{1},
\end{aligned}
$$

which implies

$$
\left\|\widetilde{p}_{k_{n}}-p_{k_{n}}^{*}\right\|_{1} \leq C\left(\left\|\gamma_{k_{n}}^{*}-\gamma_{\infty}^{*}\right\|_{0, \Gamma_{i}}+\left\|u_{k_{n}}^{*}-u_{\infty}^{*}\right\|_{1}\right) .
$$

Furthermore, it is easy to see that the problem 4.19 is a discrete version of 4.15). Hence the Cea's lemma gives

$$
\left\|p_{\infty}^{*}-\widetilde{p}_{k_{n}}\right\|_{1} \leq C \inf _{v \in V_{k_{n}}}\left\|p_{\infty}^{*}-v\right\|_{1}
$$

Now the desired result comes readily from (4.20)-(4.21), the first and last convergences in (4.18) and the construction of $V_{\infty}$.

\section{Convergence}

In this section, we shall present the main result of this paper: the sequence $\left\{\left(u_{k}^{*}, p_{k}^{*}, \gamma_{k}^{*}\right)\right\}$ generated by Algorithm 3.1 has a subsequence $\left\{\left(u_{k_{n}}^{*}, p_{k_{n}}^{*}, \gamma_{k_{n}}^{*}\right)\right\}$ converging strongly to some true solution of the problem (2.3)-(2.5). By Theorem 4.3 this reduces to verifying that $\left(u_{\infty}^{*}, p_{\infty}^{*}, \gamma_{\infty}^{*}\right)$ satisfies the system (2.3)-(2.5). For this purpose, we shall first show that two sequences of the residuals with respect to $u_{k_{n}}^{*}$ and $p_{k_{n}}^{*}$ have vanishing weak limits (see Lemma 5.2), with the help of which the limiting triplet $\left(u_{\infty}^{*}, p_{\infty}^{*}, \gamma_{\infty}^{*}\right)$ is proved to satisfy the equations (2.3) and (2.4) (see Lemma 5.3). For the variational inequality (2.5), the existing approaches for variational equations (see, e.g., [38] [41]) do not work. Instead, we shall relate it to $\xi_{k_{n}}\left(u_{k_{n}}^{*}, p_{k_{n}}^{*}, \gamma_{k_{n}}^{*}\right)$ (cf. (3.1)) through some residual of the discrete problem (2.10), then prove the error indicator tends to zero and apply a density argument (see Lemmas 5.4-5.6). This new approach is one of the key ingredients in our arguments specifically for the variational inequality (2.5) featured by the Robin inverse problem. It is also worth mentioning that the a posteriori error estimator $\eta_{k}+\xi_{k}(\mathrm{cf}$. (3.1) $)$ in the module ESTIMATE of Algorithm 3.1 is a natural consequence of the above arguments. This approach is completely different from traditional ones for boundary value problems [1] [45]. Particularly, the existing standard way is to bound the error in some norm from above by some computable quantities, while this is difficult to achieve for the state, the costate and the control variable due to the strong nonlinearity of the current inverse problem.

Before starting our proof, we state some properties of adaptively generated triangulations and the error estimator first. Let $\mathbb{T}$ be the class of all possible conforming triangulations of $\bar{\Omega}$ refined from a 
certain shape-regular initial mesh by the successive bisections [39] [43]. We call $\mathcal{T}^{\prime}$ a refinement of $\mathcal{T}$ for any $\mathcal{T}$ and $\mathcal{T}^{\prime} \in \mathbb{T}$ if $\mathcal{T}^{\prime}$ is produced from $\mathcal{T}$ by a finite number of bisections. For any triangulation sequence $\left\{\mathcal{T}_{k}\right\} \subset \mathbb{T}$ with $\mathcal{T}_{k+1}$ a refinement of $\mathcal{T}_{k}$, we define

$$
\mathcal{T}_{k}^{+}:=\bigcap_{l \geq k} \mathcal{T}_{l}, \quad \mathcal{T}_{k}^{0}:=\mathcal{T}_{k} \backslash \mathcal{T}_{k}^{+}, \quad \mathcal{F}_{k}^{+}:=\bigcap_{l \geq k} \mathcal{F}_{l}, \quad \mathcal{F}_{k}^{0}:=\mathcal{F}_{k} \backslash \mathcal{F}_{k}^{+}
$$

and

$$
\Omega_{k}^{+}:=\bigcup_{T \in \mathcal{T}_{k}^{+}} D_{T}, \quad \Omega_{k}^{0}:=\bigcup_{T \in \mathcal{T}_{k}^{0}} D_{T} .
$$

That is, $\mathcal{T}_{k}^{+}$consists of all elements not refined after the $k$-th iteration while all elements in $\mathcal{T}_{k}^{0}$ are refined at least once after the $k$-th iteration. The same is also said of faces in $\mathcal{F}_{k}^{+}$and $\mathcal{F}_{k}^{0}$. In addition, we define a mesh-size function $h_{k}: \bar{\Omega} \rightarrow \mathbb{R}^{+}$almost everywhere by $h_{k}(x)=h_{T}$ for $x$ in the interior of an element $T \in \mathcal{T}_{k}$ and $h_{k}(x)=h_{F}$ for $x$ in the relative interior of a face $F \in \mathcal{F}_{k}$. Letting $\chi_{k}^{0}$ be the characteristic function of $\Omega_{k}^{0}$, then this mesh-size function has the following property [38] [41]:

$$
\lim _{k \rightarrow \infty}\left\|h_{k} \chi_{k}^{0}\right\|_{L^{\infty}(\Omega)}=0
$$

By virtue of Theorem 4.3 and the property (5.1), we can study the convergence behavior of the maximal error indicator in the set of marked elements.

Lemma 5.1. Let $\left\{\mathcal{T}_{k}, V_{k} \times \mathscr{A}_{k},\left(u_{k}^{*}, p_{k}^{*}, \gamma_{k}^{*}\right)\right\}$ be the sequence of meshes, finite element spaces and discrete admissible sets, and discrete solutions produced by Algorithm 3.1 and $\mathcal{S}_{k}$ the set of marked elements given by (3.2). Then for the convergent subsequence $\left\{\left(u_{k_{n}}^{*}, p_{k_{n}}^{*}, \gamma_{k_{n}}^{*}\right)\right\}$ given by Theorem 4.3. there holds for the error indicator defined in (3.1) that

$$
\lim _{n \rightarrow \infty} \max _{F \in \mathcal{S}_{k_{n}}} \eta_{F, k_{n}}\left(u_{k_{n}}^{*}, p_{k_{n}}^{*}, \gamma_{k_{n}}^{*}, f, q, g\right)+\xi_{F, k_{n}}\left(u_{k_{n}}^{*}, p_{k_{n}}^{*}, \gamma_{k_{n}}^{*}\right)=0
$$

Proof. For each $k_{n}$, we denote by $\widetilde{F}$ the face in $\mathcal{S}_{k_{n}}$ with the largest error indicator. As $\widetilde{F} \in \mathcal{S}_{k_{n}}$, we know $\omega_{F} \subset \Omega_{k_{n}}^{0}$, from which and (5.1) it follows that

$$
|\widetilde{F}| \leq C\left\|h_{k_{n}}\right\|_{L^{\infty}\left(\Omega_{k_{n}}^{0}\right)}^{d-1} \rightarrow 0, \quad\left|\omega_{\widetilde{F}}\right| \leq C\left\|h_{k_{n}}\right\|_{L^{\infty}\left(\Omega_{k_{n}}^{0}\right)}^{d} \rightarrow 0 \quad \text { as } n \rightarrow \infty .
$$

By means of the stability estimate (3.3), inverse estimates and the triangle inequality, we have

$$
\begin{aligned}
\eta_{\widetilde{F}, m}^{2}\left(u_{k_{n}}^{*}, p_{k_{n}}^{*}, \gamma_{k_{n}}^{*}, f, q, g\right) \leq & C\left(\left\|u_{k_{n}}^{*}\right\|_{1, \omega_{\widetilde{F}}}^{2}+\left\|p_{k_{n}}^{*}\right\|_{1, \omega_{\widetilde{F}}}^{2}+h_{\widetilde{F}}^{2}\|f\|_{0, \omega_{\widetilde{F}}}^{2}+h_{\widetilde{F}}\|g\|_{0, \widetilde{F} \cap \Gamma_{a}}^{2}+h_{\widetilde{F}}\|q\|_{0, \widetilde{F} \cap \Gamma_{a}}^{2}\right) \\
\leq C & \left(\left\|u_{k_{n}}^{*}-u_{\infty}^{*}\right\|_{1}^{2}+\left\|u_{\infty}^{*}\right\|_{1, \omega_{\widetilde{F}}}^{2}+\left\|p_{k_{n}}^{*}-p_{\infty}^{*}\right\|_{1}^{2}+\left\|p_{\infty}^{*}\right\|_{1, \omega_{\widetilde{F}}}^{2}\right. \\
& \left.+|\widetilde{F}|^{2 /(d-1)}\|f\|_{0, \omega_{\widetilde{F}}}^{2}+|\widetilde{F}|^{1 /(d-1)}\|g\|_{0, \widetilde{F} \cap \Gamma_{a}}^{2}+|\widetilde{F}|^{1 /(d-1)}\|q\|_{0, \widetilde{F} \cap \Gamma_{a}}^{2}\right)
\end{aligned}
$$

and

$$
\begin{aligned}
& \xi_{\widetilde{F}, k_{n}}\left(u_{k_{n}}^{*}, p_{k_{n}}^{*}, \gamma_{k_{n}}^{*}\right) \leq\left(h_{\widetilde{F}}^{2}\left\|\beta \gamma_{k_{n}}^{*}\right\|_{L^{1}\left(\widetilde{F} \cap \Gamma_{i}\right)}+h_{\widetilde{F}}^{2}\left\|u_{k_{n}}^{*} p_{k_{n}}^{*}\right\|_{L^{1}\left(\widetilde{F} \cap \Gamma_{i}\right)}\right) \\
& \leq\left(h_{\widetilde{F}}^{2} \beta\left\|\gamma_{k_{n}}^{*}-\gamma_{\infty}^{*}\right\|_{L^{1}\left(\Gamma_{i}\right)}+h_{\widetilde{F}}^{2} \beta\left\|\gamma_{\infty}^{*}\right\|_{L^{1}\left(\widetilde{F} \cap \Gamma_{i}\right)}+h_{\widetilde{F}}^{2}\left\|u_{k_{n}}^{*}\right\|_{0, \widetilde{F} \cap \Gamma_{i}}\left\|p_{k_{n}}^{*}\right\|_{0, \widetilde{F} \cap \Gamma_{i}}\right) \\
& \leq C|\widetilde{F}|^{1 /(d-1)}\left(\left\|\gamma_{k_{n}}^{*}-\gamma_{\infty}^{*}\right\|_{L^{1}\left(\Gamma_{i}\right)}+\left\|\gamma_{\infty}^{*}\right\|_{L^{1}\left(\Gamma_{i}\right)}+\left(\left\|u_{k_{n}}^{*}-u_{\infty}^{*}\right\|_{1}+\left\|u_{\infty}^{*}\right\|_{1, \omega_{\widetilde{F}}}\right)\right. \\
& \times\left(\left\|p_{k_{n}}^{*}-p_{\infty}^{*}\right\|_{1}+\left\|p_{\infty}^{*}\right\|_{1, \omega_{\widetilde{F}}}\right) .
\end{aligned}
$$

Now the desired result comes from (4.18), (5.3) and the absolute continuity of $\|\cdot\|_{1}$ and $\|\cdot\|_{0, \Gamma}$ with respect to the Lebesgue measure. 
As mentioned at the beginning of this section, we now introduce two residuals with respect to $u_{k}^{*}$ and $p_{k}^{*}$ respectively as follows:

$$
\begin{gathered}
\left\langle\mathcal{R}\left(u_{k}^{*}\right), \phi\right\rangle:=\left(\alpha \boldsymbol{\nabla} u_{k}^{*}, \nabla \phi\right)+\left(\gamma_{k}^{*} u_{k}^{*}, \phi\right)_{\Gamma_{i}}-(f, \phi)-(q, \phi)_{\Gamma_{a}} \quad \forall \phi \in H^{1}(\Omega), \\
\left\langle\mathcal{R}\left(p_{k}^{*}\right), v\right\rangle:=\left(\alpha \nabla p_{k}^{*}, \nabla v\right)+\left(\gamma_{k}^{*} p_{k}^{*}, v\right)_{\Gamma_{i}}-\left(u_{k}^{*}-g, v\right)_{\Gamma_{a}} \quad \forall v \in H^{1}(\Omega) .
\end{gathered}
$$

It is easy to see from $(2.8)$ and $(2.9)$ that

$$
\left\langle\mathcal{R}\left(u_{k}^{*}\right), \phi\right\rangle=0 \quad \forall \phi \in V_{k} ; \quad\left\langle\mathcal{R}\left(p_{k}^{*}\right), v\right\rangle=0 \quad \forall v \in V_{k} .
$$

Lemma 5.2. The following convergences hold for the convergent subsequence $\left\{\left(u_{k_{n}}^{*}, p_{k_{n}}^{*}, \gamma_{k_{n}}^{*}\right)\right\}$ of $\left\{\left(u_{k}^{*}, p_{k}^{*}, \gamma_{k}^{*}\right)\right\}$ given by Theorem 4.3.

$$
\lim _{n \rightarrow \infty}\left\langle\mathcal{R}\left(u_{k_{n}}^{*}\right), \phi\right\rangle=0 \quad \text { and } \quad \lim _{n \rightarrow \infty}\left\langle\mathcal{R}\left(p_{k_{n}}^{*}\right), \phi\right\rangle=0 \quad \forall \phi \in H^{1}(\Omega) .
$$

Proof. The desired convergence can be proved in a standard way. And only the proof for the first result is given, while the second one can be done in a similar manner. Let $\left\{\left(u_{k_{n}}^{*}, p_{k_{n}}^{*}, \gamma_{k_{n}}^{*}\right)\right\}$ be the convergent subsequence given by Theorem 4.3 . For notational convenience, the counter $k_{n}$ is replaced with $m$. We write $I_{m}$ and $I_{m}^{s z}$ for the Lagrange and Scott-Zhang interpolation operators respectively associated with $V_{k}$ [40] and use the orthogonality (5.4) and the definition (3.1) to proceed for $m>l$ and any $\psi \in C^{\infty}(\bar{\Omega})$ :

$$
\begin{aligned}
& \left|\left\langle\mathcal{R}\left(u_{m}^{*}\right), \psi\right\rangle\right|=\left|\left\langle\mathcal{R}\left(u_{m}^{*}\right), \psi-I_{m} \psi\right\rangle\right|=\left|\left\langle\mathcal{R}\left(u_{m}^{*}\right), \psi-I_{m} \psi-I_{m}^{s z}\left(\psi-I_{m} \psi\right)\right\rangle\right| \\
\leq & C \sum_{F \in \mathcal{F}_{m}} \eta_{F, m, 1}\left(u_{m}^{*}, \gamma_{m}^{*}, f, q\right)\left\|\psi-I_{m} \psi\right\|_{1, \cup D_{T}} \\
= & C\left(\sum_{F \in \mathcal{F}_{m} \backslash \mathcal{F}_{l}^{+}} \eta_{F, m, 1}\left(u_{m}^{*}, \gamma_{m}^{*}, f, q\right)\left\|\psi-I_{m} \psi\right\|_{1, \cup D_{T}}+\sum_{F \in \mathcal{F}_{l}^{+}} \eta_{F, m, 1}\left(u_{m}^{*}, \gamma_{m}^{*}, f, q\right)\left\|\psi-I_{m} \psi\right\|_{1, \cup D_{T}}\right),
\end{aligned}
$$

where $\cup D_{T}$ is the union of $D_{T}$ with $T \in \omega_{F}$. In the third inequality, we also use the definition of $\left\langle\mathcal{R}\left(u_{m}^{*}\right), \cdot\right\rangle$, elementwise integration by parts, error estimates for $I_{m}^{s z}$ [40] and the Cauchy-Schwarz inequality. Noting Lemma 3.1 and the stability estimate (2.11) we have

$$
\left(\sum_{F \in \mathcal{F}_{m} \backslash \mathcal{F}_{l}^{+}} \eta_{F, m, 1}^{2}\left(u_{m}^{*}, \gamma_{m}^{*}, f, q\right)\right)^{1 / 2} \leq C
$$

and then by the error estimate for $I_{m}$ we are further led to

$$
\left|\left\langle\mathcal{R}\left(u_{m}^{*}\right), \psi\right\rangle\right| \leq C_{1}\left\|h_{l}\right\|_{L^{\infty}\left(\Omega_{l}^{0}\right)}\|\psi\|_{2}+C_{2}\left(\sum_{F \in \mathcal{F}_{l}^{+}} \eta_{F, m, 1}^{2}\left(u_{m}^{*}, \gamma_{m}^{*}, f, q\right)\right)^{1 / 2}\|\psi\|_{2} .
$$

Due to (5.1), for any given $\varepsilon>0$ we have $C_{1}\left\|h_{l}\right\|_{L^{\infty}\left(\Omega_{l}^{0}\right)}\|\phi\|_{2}<\varepsilon$ for sufficiently large $l$. Moreover, the inclusions that $\mathcal{F}_{l}^{+} \subset \mathcal{F}_{m}^{+} \subset \mathcal{F}_{m}$ for $m>l$ and the marking property (cf. (3.2)) imply that

$$
\begin{aligned}
\left(\sum_{F \in \mathcal{F}_{l}^{+}} \eta_{F, m, 1}^{2}\left(u_{m}^{*}, \gamma_{m}^{*}, f, q\right)\right)^{1 / 2} & \leq \sqrt{\left|\mathcal{F}_{l}^{+}\right|} \max _{F \in \mathcal{F}_{l}^{+}} \eta_{F, m, 1}\left(u_{m}^{*}, \gamma_{m}^{*}, f, q\right) \\
& \leq \sqrt{\left|\mathcal{F}_{l}^{+}\right|} \max _{F \in \mathcal{S}_{m}} \eta_{F, m}\left(u_{m}^{*}, p_{m}^{*}, \gamma_{m}^{*}, f, q, g\right)+\xi_{F, m}\left(u_{m}^{*}, p_{m}^{*}, \gamma_{m}^{*}\right)
\end{aligned}
$$

By Lemma 5.1, we can choose $M \geq l$ for some fixed $l$ such that the following estimate holds for $m \geq M$ :

$$
C_{2}\left(\sum_{F \in \mathcal{F}_{l}^{+}} \eta_{F, m, 1}^{2}\left(u_{m}^{*}, \gamma_{m}^{*}, f, q\right)\right)^{1 / 2}\|\psi\|_{2}<\varepsilon
$$


This proves

$$
\lim _{m \rightarrow \infty}\left\langle\mathcal{R}\left(u_{m}^{*}\right), \psi\right\rangle=0 \quad \forall \psi \in C^{\infty}(\bar{\Omega}),
$$

which, in combination with the density of $C^{\infty}(\bar{\Omega})$ in $H^{1}(\Omega)$, yields the first vanishing limit.

Lemma 5.3. The solution $\left(u_{\infty}^{*}, p_{\infty}^{*}, \gamma_{\infty}^{*}\right)$ of the problem (4.14)-(4.16) also satisfies the variational problems (2.3) and (2.4), i.e.,

$$
\begin{aligned}
& \left(\alpha \nabla u_{\infty}^{*}, \nabla \phi\right)+\left(\gamma_{\infty}^{*} u_{\infty}^{*}, \phi\right)_{\Gamma_{i}}=(f, \phi)+(q, \phi)_{\Gamma_{a}} \quad \forall \phi \in H^{1}(\Omega) \\
& \left(\alpha \nabla p_{\infty}^{*}, \nabla v\right)+\left(\gamma_{\infty}^{*} p_{\infty}^{*}, v\right)_{\Gamma_{i}}=\left(u_{\infty}^{*}-g, v\right)_{\Gamma_{a}} \quad \forall v \in H^{1}(\Omega) .
\end{aligned}
$$

Proof. Let $\left\{\left(u_{k_{n}}^{*}, p_{k_{n}}^{*}, \gamma_{k_{n}}^{*}\right)\right\}$ be the convergent subsequence given by Theorem 4.3 . For any $\phi \in H^{1}(\Omega)$, we can easily deduce

$$
\begin{aligned}
& \left|\left(\alpha \nabla u_{\infty}^{*}, \nabla \phi\right)+\left(\gamma_{\infty}^{*} u_{\infty}^{*}, \phi\right)_{\Gamma_{i}}-(f, \phi)-(q, \phi)_{\Gamma_{a}}\right| \\
= & \left|\left(\alpha \nabla\left(u_{\infty}^{*}-u_{k_{n}}^{*}\right), \nabla \phi\right)+\left(\gamma_{\infty}^{*} u_{\infty}^{*}-\gamma_{k_{n}}^{*} u_{k_{n}}^{*}, \phi\right)_{\Gamma_{i}}+\left\langle\mathcal{R}\left(u_{k_{n}}^{*}\right), \phi\right\rangle\right| \\
\leq & \alpha_{2}\left\|u_{\infty}^{*}-u_{k_{n}}^{*}\right\|_{1}\|\phi\|_{1}+\left|\left(\gamma_{\infty}^{*} u_{\infty}^{*}-\gamma_{k_{n}}^{*} u_{k_{n}}^{*}, \phi\right)_{\Gamma_{i}}\right|+\left|\left\langle\mathcal{R}\left(u_{k_{n}}^{*}\right), \phi\right\rangle\right| .
\end{aligned}
$$

The second term above is further estimated by the generalized Hölder inequality, the Sobolev embedding theorem and the trace theorem

$$
\begin{aligned}
\left|\left(\gamma_{\infty}^{*} u_{\infty}^{*}-\gamma_{k_{n}}^{*} u_{k_{n}}^{*}, \phi\right)_{\Gamma_{i}}\right| & =\left|\left(\left(\gamma_{\infty}^{*}-\gamma_{k_{n}}^{*}\right) u_{\infty}^{*}, \phi\right)_{\Gamma_{i}}+\left(\gamma_{k_{n}}^{*}\left(u_{\infty}^{*}-u_{k_{n}}^{*}\right), \phi\right)_{\Gamma_{i}}\right| \\
& \leq\left\|\gamma_{\infty}^{*}-\gamma_{k_{n}}^{*}\right\|_{0, \Gamma_{i}}\left\|u_{\infty}^{*}\right\|_{L^{4}\left(\Gamma_{i}\right)}\|\phi\|_{L^{4}\left(\Gamma_{i}\right)}+c_{1}\left\|u_{\infty}^{*}-u_{k_{n}}^{*}\right\|_{0, \Gamma_{i}}\|\phi\|_{0, \Gamma_{i}} \\
& \leq C\left(\left\|\gamma_{\infty}^{*}-\gamma_{k_{n}}^{*}\right\|_{0, \Gamma_{i}}\left\|u_{\infty}^{*}\right\|_{1}+c_{1}\left\|u_{\infty}^{*}-u_{k_{n}}^{*}\right\|_{1}\right)\|\phi\|_{1} .
\end{aligned}
$$

Theorem 4.3, the stability (4.17) and Lemma 5.2 imply that the three terms in the right-hand side of (5.8) all tend to zero as $n$ goes to infinity, from which we conclude the first equation (5.6).

As for the second equation (5.7), it is not difficult to proceed as above for any $v \in H^{1}(\Omega)$ that

$$
\begin{aligned}
& \left|\left(\alpha \nabla p_{\infty}^{*}, \nabla v\right)+\left(\gamma_{\infty}^{*} p_{\infty}^{*}, v\right)_{\Gamma_{i}}-\left(u_{\infty}^{*}-g, v\right)_{\Gamma_{a}}\right| \\
= & \left|\left(\alpha \nabla\left(p_{\infty}^{*}-p_{k_{n}}^{*}\right), \nabla v\right)+\left(\gamma_{\infty}^{*} p_{\infty}^{*}-\gamma_{k_{n}}^{*} p_{k_{n}}^{*}, v\right)_{\Gamma_{i}}-\left(u_{\infty}^{*}-u_{k_{n}}^{*}, v\right)_{\Gamma_{a}}+\left\langle\mathcal{R}\left(p_{k_{n}}^{*}\right), v\right\rangle\right| \\
\leq & \alpha_{2}\left\|p_{\infty}^{*}-p_{k_{n}}^{*}\right\|_{1}\|v\|_{1}+\left|\left(\gamma_{\infty}^{*} p_{\infty}^{*}-\gamma_{k_{n}}^{*} p_{k_{n}}^{*}, v\right)_{\Gamma_{i}}\right|+\left\|u_{\infty}^{*}-u_{k_{n}}^{*}\right\|_{0, \Gamma_{a}}\|v\|_{1}+\left|\left\langle\mathcal{R}\left(p_{k_{n}}^{*}\right), v\right\rangle\right| .
\end{aligned}
$$

Then the proof is finished by using Theorem 4.3, Lemma 5.2 and same argument as for (5.8).

Next we turn our attention to the claim: the variational inequality 2.5 holds for the limiting triplet $\left(u_{\infty}^{*}, p_{\infty}^{*}, \gamma_{\infty}^{*}\right)$. As in the proof of Lemma 5.2, our approach still lies in a density argument. Therefore, we need the following density result with respect to the admissible set $\mathscr{A}$.

Lemma 5.4. Suppose $\Gamma_{i}$ is split in $L$ open faces/edges $\Gamma_{i, 1}, \cdots, \Gamma_{i, L}$, then $\widetilde{\mathscr{A}}=\left\{\lambda \in \mathscr{A} \cap C\left(\bar{\Gamma}_{i}\right) \mid \lambda \in\right.$ $\left.C^{\infty}\left(\bar{\Gamma}_{i, l}\right), 1 \leq l \leq L\right\}$ is weakly-* dense in $\mathscr{A}$ with respect to $L^{\infty}\left(\Gamma_{i}\right)$.

Proof. We only focus on $d=3$ as the arguments for the two dimensional case are similar. Let us first assume that $\Gamma_{i}$ is the intersection of the whole boundary $\Gamma$ with a plane in $\mathbb{R}^{2}$, i.e. $L=1$. For any $\lambda \in \mathscr{A}$, we define an extension of $\widetilde{\lambda}$ in $\mathbb{R}^{2}$ as $\widetilde{\lambda}:=\lambda$ in $\Gamma_{i} ; \widetilde{\lambda}:=c_{0}$ in $\Gamma_{i, 3 \epsilon_{0} / 2} \backslash \Gamma_{i} ; \widetilde{\lambda}:=0$ in $\Gamma_{i, 3 \epsilon_{0}} \backslash \Gamma_{i, 3 \epsilon_{0} / 2}$, where $\epsilon_{0}>0$ is a positive constant and $\Gamma_{i, \delta}:=\left\{\boldsymbol{x} \in \mathbb{R}^{2} \mid d\left(x, \bar{\Gamma}_{i}\right)<\delta\right\}$ with $d\left(\boldsymbol{x}, \bar{\Gamma}_{i}\right)$ denoting the distance between $\boldsymbol{x}$ and $\bar{\Gamma}_{i}$ and $\delta>0$. Recall $c_{0}$ is the lower bound of the Robin coefficient $\gamma$. Then we make use of the standard mollifier $\rho(\boldsymbol{x})$ in $\mathbb{R}^{2}$ to construct a sequence $\left\{\lambda_{n}\right\}_{n \geq 0} \subset C^{\infty}\left(\bar{\Gamma}_{i}\right)$ with $\lambda_{n}=\left.\left(\epsilon_{n}^{-2} \rho\left(\boldsymbol{x} / \epsilon_{n}\right) * \widetilde{\lambda}\right)\right|_{\bar{\Gamma}_{i}}$, where $\left\{\epsilon_{n}\right\}$ is a positive sequence of numbers decreasing to zero. Since $\lambda_{n} \rightarrow \lambda$ a.e. in $\Gamma_{i}$ as $\epsilon_{n} \rightarrow 0^{+}$(cf. [17]) and $\sup _{n}\left\|\lambda_{n}\right\|_{L^{\infty}\left(\Gamma_{i}\right)} \leq c_{1}$, the upper bound of the Robin 
coefficient $\gamma$, the Dominated Convergence Theorem admits $\lambda_{n} \rightarrow \lambda$ weakly-* in $L^{\infty}\left(\Gamma_{i}\right)$. Moreover by the construction of $\left\{\lambda_{n}\right\}$, we find $c_{0} \leq \lambda_{n} \leq c_{1}$ a.e. in $\Gamma_{i}$ for all $n$.

For the general case that $\bar{\Gamma}_{i}=\cup_{l=1}^{L} \bar{\Gamma}_{i, l}$, we extend any $\lambda \in \mathscr{A}$ on the whole boundary $\Gamma$ such that $\bar{\lambda}:=\lambda$ on $\Gamma_{i}$ and $\bar{\lambda}:=c_{0}$ otherwise. As the boundary $\Gamma$ is closed and Lipschitz continuous, there exist finite open cubes $\left\{U_{j}\right\}_{1 \leq j \leq J}$ covering $\Gamma$ and a corresponding Lipschitz continuous function sequence $\left\{\phi_{j}\right\}_{1 \leq j \leq J}$ of 2 variables such that $\Gamma \cap U_{j}$ is the graph of $\phi_{j}$ defined on some open square $S_{j}$ in $\mathbb{R}^{2}$. Arguing as above, on each $\Gamma \cap U_{j}$ we obtain a sequence $\left\{\bar{\lambda}_{j, n}\left(\boldsymbol{x}, \phi_{j}(\boldsymbol{x})\right)\right\} \subset C^{\infty}\left(\bar{S}_{j}\right)$ satisfying that $\bar{\lambda}_{j, n} \rightarrow \bar{\lambda}$ weakly-* in $L^{\infty}\left(\Gamma \cap U_{j}\right)$ when $n \rightarrow \infty$ and $c_{0} \leq \bar{\lambda}_{j, n} \leq c_{1}$ a.e. in $\Gamma \cap U_{j}$. Then using the partition of unity $\left\{\psi_{j}\right\}_{1 \leq j \leq J}$ subordinate to $\left\{U_{j}\right\}$ and noting $\phi_{j}$ is smooth when $\left(\boldsymbol{x}, \phi_{j}(\boldsymbol{x})\right)$ in some $\bar{\Gamma}_{i, l}, \lambda_{n}:=\left.\left(\sum_{j=1}^{J} \psi_{j} \bar{\lambda}_{j, n}\right)\right|_{\bar{\Gamma}_{i}} \in \widetilde{\mathscr{A}}$ and $\lambda_{n} \rightarrow \lambda$ weakly-* in $L^{\infty}\left(\Gamma_{i}\right)$, which completes the proof.

With Lemma 5.4 in hand, we first prove the result in $\widetilde{\mathscr{A}}$ and then extend to $\mathscr{A}$.

Lemma 5.5. The solution $\left(u_{\infty}^{*}, p_{\infty}^{*}, \gamma_{\infty}^{*}\right)$ of the problem 4.14 (4.16) satisfies

$$
\left(\beta \gamma_{\infty}^{*}-u_{\infty}^{*} p_{\infty}^{*}, \mu-\gamma_{\infty}^{*}\right)_{\Gamma_{i}} \geq 0 \quad \forall \mu \in \widetilde{\mathscr{A}}
$$

Proof. As in the proof of Lemma 5.2, we still denote the convergent subsequence $\left\{\left(u_{k_{n}}^{*}, p_{k_{n}}^{*}, \gamma_{k_{n}}^{*}\right)\right\}$ given in Theorem 4.3 by $\left\{\left(u_{m}^{*}, p_{m}^{*}, \gamma_{m}^{*}\right)\right\}$. Invoking the Lagrange interpolation operator $\mathscr{I}_{m}$ associated with $V_{m, \Gamma_{i}}$, we note $\mathscr{I}_{m} \mu \in \mathscr{A}_{m}$ for any $\mu \in \widetilde{\mathscr{A}}$. Then the variational inequality (2.10) allows

$$
\begin{aligned}
\left(\beta \gamma_{m}^{*}-u_{m}^{*} p_{m}^{*}, \mu-\gamma_{m}^{*}\right)_{\Gamma_{i}} & =\left(\beta \gamma_{m}^{*}-u_{m}^{*} p_{m}^{*}, \mu-\mathscr{I}_{m} \mu\right)_{\Gamma_{i}}+\left(\beta \gamma_{m}^{*}-u_{m}^{*} p_{m}^{*}, \mathscr{I}_{m} \mu-\gamma_{m}^{*}\right)_{\Gamma_{i}} \\
& \geq\left(\beta \gamma_{m}^{*}-u_{m}^{*} p_{m}^{*}, \mu-\mathscr{I}_{m} \mu\right)_{\Gamma_{i}} \quad \forall \mu \in \widetilde{\mathscr{A}} .
\end{aligned}
$$

By the error estimate of $\mathscr{I}_{m}$ [15] and the definition of $\xi_{m}$ in (3.1) we know for $m>l$

$$
\begin{aligned}
\left|\left(\beta \gamma_{m}^{*}-u_{m}^{*} p_{m}^{*}, \mu-\mathscr{I}_{m} \mu\right)_{\Gamma_{i}}\right| & \leq \sum_{F \in \mathcal{F}_{m}\left(\Gamma_{i}\right)}\left\|\beta \gamma_{m}^{*}-u_{m}^{*} p_{m}^{*}\right\|_{L^{1}(F)}\left\|\mu-\mathscr{I}_{m} \mu\right\|_{L^{\infty}(F)} \\
& \leq C \sum_{F \in \mathcal{F}_{m}\left(\Gamma_{i}\right)} \xi_{F, m}\left(u_{m}^{*}, p_{m}^{*}, \gamma_{m}^{*}\right)\|\mu\|_{2, \infty, \Gamma_{i}} \\
& =C\left(\xi_{m}\left(u_{m}^{*}, p_{m}^{*}, \gamma_{m}^{*}, \mathcal{F}_{m} \backslash \mathcal{F}_{l}^{+}\right)+\xi_{m}\left(u_{m}^{*}, p_{m}^{*}, \gamma_{m}^{*}, \mathcal{F}_{l}^{+}\right)\right)\|\mu\|_{2, \infty, \Gamma_{i}} .
\end{aligned}
$$

By the definition of $\xi_{m}$, the constraint of $\gamma_{m}^{*}$ and the stability estimate (2.11), we further come to

$$
\begin{aligned}
\xi_{m}\left(u_{m}^{*}, p_{m}^{*}, \gamma_{m}^{*}, \mathcal{F}_{m} \backslash \mathcal{F}_{l}^{+}\right) & \leq C\left\|h_{l}\right\|_{L^{\infty}\left(\Omega_{l}^{0}\right)}^{2}\left\|\gamma_{m}^{*}-u_{m}^{*} p_{m}^{*}\right\|_{L^{1}\left(\Gamma_{i}\right)} \\
& \leq C\left\|h_{l}\right\|_{L^{\infty}\left(\Omega_{l}^{0}\right)}^{2}\left(\left\|\gamma_{m}^{*}\right\|_{L^{1}\left(\Gamma_{i}\right)}+\left\|u_{m}^{*}\right\|_{L^{2}\left(\Gamma_{i}\right)}\left\|p_{m}^{*}\right\|_{L^{2}\left(\Gamma_{i}\right)}\right) \\
& \leq C\left\|h_{l}\right\|_{L^{\infty}\left(\Omega_{l}^{0}\right)}^{2}\left(c_{1}\left|\Gamma_{i}\right|+\left\|u_{m}^{*}\right\|_{1}\left\|p_{m}^{*}\right\|_{1}\right) \leq C\left\|h_{l}\right\|_{L^{\infty}\left(\Omega_{l}^{0}\right)}^{2}
\end{aligned}
$$

Therefore,

$$
\left|\left(\beta \gamma_{m}^{*}-u_{m}^{*} p_{m}^{*}, \mu-\mathscr{I}_{m} \mu\right)_{\Gamma_{i}}\right| \leq C_{3}\left\|h_{l}\right\|_{L^{\infty}\left(\Omega_{l}^{0}\right)}^{2}\|\mu\|_{2, \infty, \Gamma_{i}}+C_{4} \xi_{m}\left(u_{m}^{*}, p_{m}^{*}, \gamma_{m}^{*}, \mathcal{F}_{l}^{+}\right)\|\mu\|_{2, \infty, \Gamma_{i}} .
$$

Now noting (5.1), we find that for any given $\varepsilon>0$ there exists a large $l$ such that

$$
C_{3}\left\|h_{l}\right\|_{L^{\infty}\left(\Omega_{l}^{0}\right)}^{2}\|\mu\|_{2, \infty, \Gamma_{i}}<\varepsilon .
$$

Since $\mathcal{F}_{l}^{+} \cap \mathcal{S}_{m}=\emptyset$ the condition $(3.2)$ in the module MARK of Algorithm 3.1 admits

$$
\begin{aligned}
\xi_{m}\left(u_{m}^{*}, p_{m}^{*}, \gamma_{m}^{*}, \mathcal{F}_{l}^{+}\right) & \leq\left|\mathcal{F}_{l}^{+}\right| \max _{F \in \mathcal{F}_{l}^{+} \cap \mathcal{F}_{m}\left(\Gamma_{i}\right)} \xi_{F, m}\left(u_{m}^{*}, p_{m}^{*}, \gamma_{m}^{*}\right) \\
& \leq\left|\mathcal{F}_{l}^{+}\right| \max _{F \in \mathcal{S}_{m}} \eta_{F, m}\left(u_{m}^{*}, p_{m}^{*}, \gamma_{m}^{*}, q, g\right)+\xi_{F, m}\left(u_{m}^{*}, p_{m}^{*}, \gamma_{m}^{*}\right) .
\end{aligned}
$$


Then using Lemma 5.1, we can choose $M \geq l$ such that

$$
C_{4} \xi_{m}\left(u_{m}^{*}, p_{m}^{*}, \gamma_{m}^{*}, \mathcal{F}_{l}^{+}\right)\|\mu\|_{2, \infty, \Gamma_{i}}<\varepsilon
$$

for $m \geq M$. Summarizing (5.11)-(5.13) gives

$$
\left(\beta \gamma_{m}^{*}-u_{m}^{*} p_{m}^{*}, \mu-\mathscr{I}_{m} \mu\right)_{\Gamma_{i}} \rightarrow 0 \quad \text { as } m \rightarrow \infty .
$$

On the other hand, recalling some elementary identities

$$
\begin{gathered}
\left(u_{m}^{*} p_{m}^{*}-u_{\infty}^{*} p_{\infty}^{*}, \mu\right)_{\Gamma_{i}}=\left(\left(u_{m}^{*}-u_{\infty}^{*}\right) p_{m}^{*}+\left(p_{m}^{*}-p_{\infty}^{*}\right) u_{\infty}^{*}, \mu\right)_{\Gamma_{i}}, \\
\left(u_{m}^{*} p_{m}^{*}, \gamma_{m}^{*}\right)_{\Gamma_{i}}-\left(u_{\infty}^{*} p_{\infty}^{*}, \gamma_{\infty}^{*}\right)_{\Gamma_{i}}=\left(\left(u_{m}^{*} p_{m}^{*}-u_{\infty}^{*} p_{\infty}^{*}\right), \gamma_{m}^{*}\right)_{\Gamma_{i}}+\left(u_{\infty}^{*} p_{\infty}^{*}, \gamma_{m}^{*}-\gamma_{\infty}^{*}\right)_{\Gamma_{i}},
\end{gathered}
$$

we know from the generalized Hölder inequality, the stability estimates (2.11), (4.17) and Theorem 4.3 that

$$
\lim _{m \rightarrow \infty}\left(\beta \gamma_{m}^{*}-u_{m}^{*} p_{m}^{*}, \mu-\gamma_{m}^{*}\right)_{\Gamma_{i}}=\left(\beta \gamma_{\infty}^{*}-u_{\infty}^{*} p_{\infty}^{*}, \mu-\gamma_{\infty}^{*}\right)_{\Gamma_{i}} \quad \forall \mu \in \widetilde{\mathscr{A}}
$$

The proof is concluded by a combination of (5.14) and (5.15) in (5.10).

Lemma 5.6. The solution $\left(u_{\infty}^{*}, p_{\infty}^{*}, \gamma_{\infty}^{*}\right)$ of the problem (4.14)-(4.16) satisfies the variational inequality 2.5, i.e.,

$$
\left(\beta \gamma_{\infty}^{*}-u_{\infty}^{*} p_{\infty}^{*}, \lambda-\gamma_{\infty}^{*}\right)_{\Gamma_{i}} \geq 0 \quad \forall \lambda \in \mathscr{A}
$$

Proof. We argue by contradiction. If (5.16) fails, then there exists some $\nu \in \mathscr{A}$ such that

$$
\left(\beta \gamma_{\infty}^{*}-u_{\infty}^{*} p_{\infty}^{*}, \nu-\gamma_{\infty}^{*}\right)_{\Gamma_{i}}<0 .
$$

Lemma 5.4 implies for any $\varepsilon>0$ there exists a $\mu \in \widetilde{\mathscr{A}}$ such that

$$
\begin{aligned}
\left(\beta \gamma_{\infty}^{*}-u_{\infty}^{*} p_{\infty}^{*}, \mu-\gamma_{\infty}^{*}\right)_{\Gamma_{i}} & =\left(\beta \gamma_{\infty}^{*}-u_{\infty}^{*} p_{\infty}^{*}, \mu-\nu\right)_{\Gamma_{i}}+\left(\beta \gamma_{\infty}^{*}-u_{\infty}^{*} p_{\infty}^{*}, \nu-\gamma_{\infty}^{*}\right) \Gamma_{i} \\
& <\varepsilon+\left(\beta \gamma_{\infty}^{*}-u_{\infty}^{*} p_{\infty}^{*}, \nu-\gamma_{\infty}^{*}\right) \Gamma_{i} .
\end{aligned}
$$

It follows from (5.17) that when $\varepsilon \leq \varepsilon_{0}$ for some $\varepsilon_{0}>0$,

$$
\left(\beta \gamma_{\infty}^{*}-u_{\infty}^{*} p_{\infty}^{*}, \mu-\gamma_{\infty}^{*}\right)_{\Gamma_{i}}<0 .
$$

This contradicts Lemma 5.5.

Remark 5.1. As can be seen from the proof of Lemma 5.2 and Lemma 5.5, the residuals $\left\langle\mathcal{R}\left(u_{m}^{*}\right), \phi\right\rangle$ and $\left\langle\mathcal{R}\left(p_{m}^{*}\right), \phi\right\rangle$ associated with $(2.8)$ and $(2.9)$ are bounded by

$$
\left|\left\langle\mathcal{R}\left(u_{m}^{*}\right), \phi\right\rangle\right|+\left|\left\langle\mathcal{R}\left(p_{m}^{*}\right), \phi\right\rangle\right| \leq C \eta_{m}\left(u_{m}^{*}, p_{m}^{*}, \gamma_{m}^{*}, f, q, g\right)\|\phi\|_{1} \quad \forall \phi \in H^{1}(\Omega)
$$

and the residual $\left(\beta \gamma_{m}^{*}-u_{m}^{*} p_{m}^{*}, \mu-\gamma_{m}^{*}\right)_{\Gamma_{i}}$ with respect to the variational inequality (2.10) leads to

$$
\left|\left(\beta \gamma_{m}^{*}-u_{m}^{*} p_{m}^{*}, \mu-\mathscr{I}_{m} \mu\right)_{\Gamma_{i}}\right| \leq C \xi_{m}\left(u_{m}^{*}, p_{m}^{*}, \gamma_{m}^{*}\right)\|\mu\|_{2, \infty, \Gamma_{i}} \quad \forall \mu \in \widetilde{\mathscr{A}} .
$$

The terms $\eta_{m}$ and $\xi_{m}$ (see (3.1) for definition) in the right-hand side of the above two inequalities only depend on the discrete solutions, the mesh size and the given data. Consequently, we use $\eta_{m}+\xi_{m}$ as the estimator in Algorithm 3.1. More importantly, the proofs of the above lemmas show that the estimator is sufficient for convergence of adaptive algorithm although it does not provide an upper bound of the error $\left\|u^{*}-u_{k}^{*}\right\|_{1},\left\|p^{*}-p_{k}^{*}\right\|_{1}$ and $\left\|\gamma^{*}-\gamma_{k}^{*}\right\|_{0, \Gamma_{i}}$. In terms of the a posteriori error analysis, this is quite different from the existing works for direct partial differential equations.

Finally, by virtue of Theorem 4.3 . Lemma 5.3 and Lemma 5.6 we present the main result of the current work.

Theorem 5.1. The sequence of discrete solutions $\left\{\left(u_{k}^{*}, p_{k}^{*}, \gamma_{k}^{*}\right)\right\}$ produced by Algorithm 3.1 has a subsequence converging to a solution $\left(u^{*}, p^{*}, \gamma^{*}\right)$ of the problem 2.3)-2.5) in the following sense:

$$
\left\|u_{k_{n}}^{*}-u^{*}\right\|_{1} \rightarrow 0, \quad\left\|p_{k_{n}}^{*}-p^{*}\right\|_{1} \rightarrow 0, \quad\left\|\gamma_{k_{n}}^{*}-\gamma^{*}\right\|_{0, \Gamma_{i}} \rightarrow 0 \quad \text { as } n \rightarrow \infty
$$




\section{$6 \quad$ Numerical experiments}

In this section, we shall provide some numerical experiments to verify the effectiveness of our proposed adaptive finite element Algorithm 3.1. In all the experiments, the general requirement (3.2) in the module MARK is represented by a specific maximum strategy (see Remark 3.1), i.e., mark a subset $\mathcal{S}_{k} \subset \mathcal{F}_{k}$ such that

$$
\eta_{F, k}\left(u_{k}^{*}, p_{k}^{*}, \gamma_{k}^{*}, f, q, g\right)+\xi_{F, k}\left(u_{k}^{*}, p_{k}^{*}, \gamma_{k}^{*}\right) \geq 0.5 \max _{F \in \mathcal{F}_{k}}\left(\eta_{F, k}\left(u_{k}^{*}, p_{k}^{*}, \gamma_{k}^{*}, f, q, g\right)+\xi_{F, k}\left(u_{k}^{*}, p_{k}^{*}, \gamma_{k}^{*}\right)\right)
$$

for any $F \in \mathcal{S}_{k}$. The resulting discrete nonlinear optimization problems $(2.6)-(2.7)$ are solved by a conjugate gradient method formulated in 31 and the initial guess of the Robin coefficient for reconstruction on $\Gamma_{i}$ is set to be constants 0.1 and 0.2 everywhere for Examples 1 and 2 respectively. These are very poor initial guesses for the concerned nonlinear inverse problem.

We will mainly focus on the difficulties arising from the challenging nature of two different types of Robin coefficients. To make our examples more practical and reasonable, the true solution $u(\boldsymbol{x})$ of the diffusion system (2.3) is assumed to be unknown in advance and is calculated in a very fine mesh under the boundary conditions $(1.2)$ on $\Gamma_{a}$ and $\Gamma_{i}$, with coefficients $\alpha=1$ and $f=0$ in $\Omega$, and $q=1$ on $\Gamma_{a}$. In applications, the boundary data $g$ on $\Gamma_{a}$ is experimentally measured and thus inevitably contaminated by measurement errors. In our examples, the simulated noisy data is synthesized as follows:

$$
g(\boldsymbol{x})=u(\boldsymbol{x})+\delta u(\boldsymbol{x}) \operatorname{rand}(\boldsymbol{x}) \text { on } \Gamma_{a},
$$

where $u(\boldsymbol{x})$ denotes the true solution, $\delta$ represents the noise level and is set to be $1 \%$, and $\operatorname{rand}(\boldsymbol{x})$ is a uniformly distributed random function in the range between -1 and 1 .

The computational domain is designed to be an open domain lying between two circles centred at the origin, with its outer boundary $\Gamma_{a}$ being a circle with radius 2 and its inner boundary $\Gamma_{i}$ being a unit circle. The outer boundary $\Gamma_{a}$ is accessible while the inner boundary $\Gamma_{i}$ is inaccessible.

For ease of visualization, the plot of the Robin coefficient on the inner boundary $\Gamma_{i}$ is parametrized in the order of left, bottom, right and top by its arc length so that the Robin coefficients can be represented by a function of arc length in one dimension.

Example 1 (Robin coefficient with sharp spike) The true distribution of the Robin coefficient has

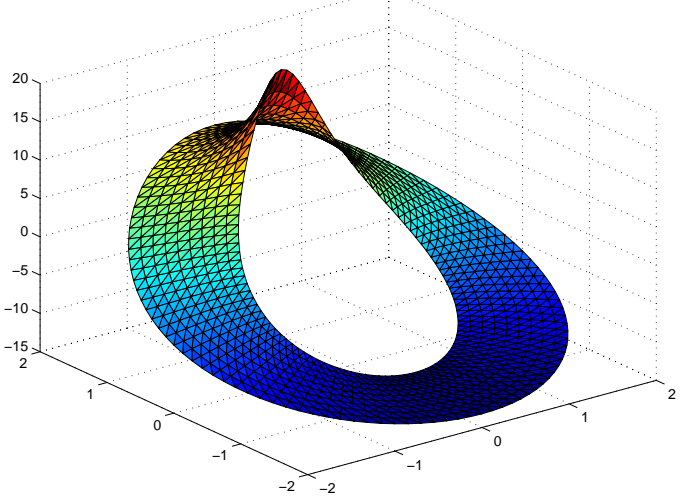

(a)

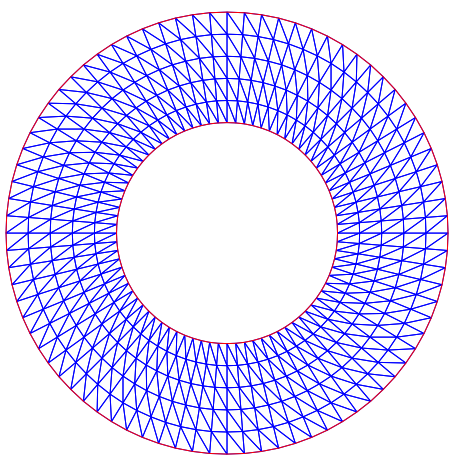

(b)

Figure 1: True solution $u$ (left) and the initial mesh with 480 nodes (right) in Example 1. 


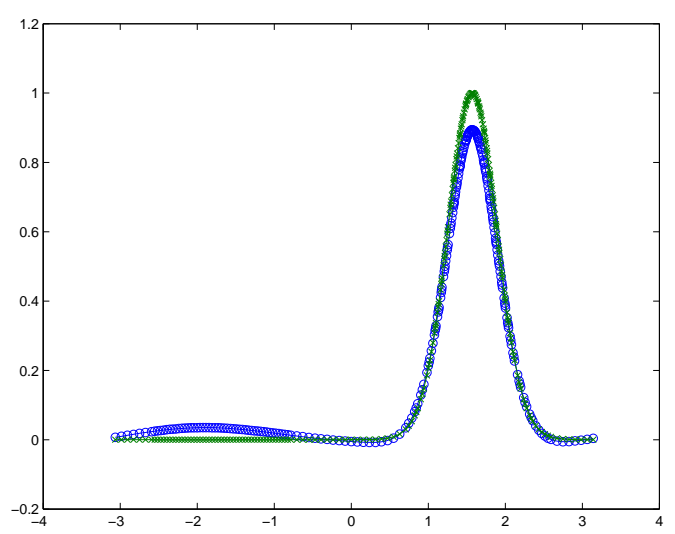

(a) $L^{2}$-norm error of $\gamma=6.8835 e-03, \beta=10^{-5}$

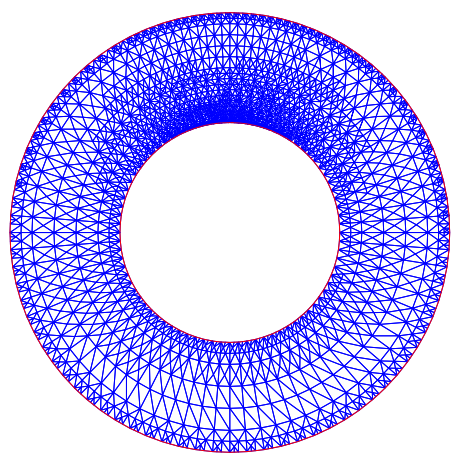

(b) Final mesh with 2406 nodes by adaptive Algorithm 3.1

Figure 2: Exact and numerically reconstructed Robin coefficients (left) and adaptively generated final mesh (right) in Example 1.

a sharp spike at the point $(0,1)$ and is given by

$$
\gamma(x, y)=\exp \left(-10\left(x^{2}+(y-1)^{2}\right)\right)
$$

restricted on the inner boundary $\Gamma_{i}$.

The true solution $u$ is shown in Figure 1(a). With Algorithm 3.1 starting from the initial mesh with 480 nodes in Figure 1(b), the reconstructed Robin coefficient (blue) approximates the exact one (green) reasonably well as shown in Figure 2(a) and the corresponding adaptively generated mesh is displayed in Figure 2(b). We observe that the reconstructed coefficient has well captured the location and height of the sharp spike. In addition, mesh refinements are correctly centered around the upper point of the interior boundary due to the spike point of the Robin coefficient.

Example 2 (Discontinuous Robin coefficient) In this example, the true Robin coefficient is set to

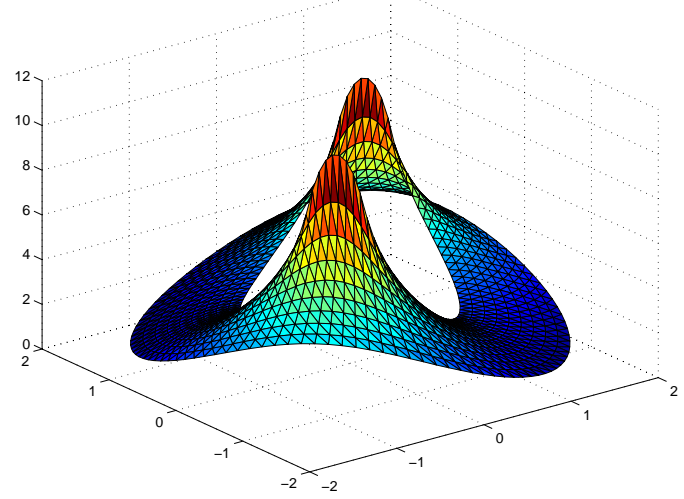

(a)

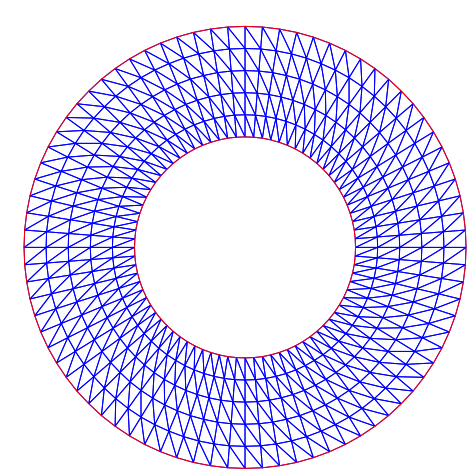

(b)

Figure 3: True solution $u$ (left) and the initial mesh with 480 nodes (right) in Example 2. 


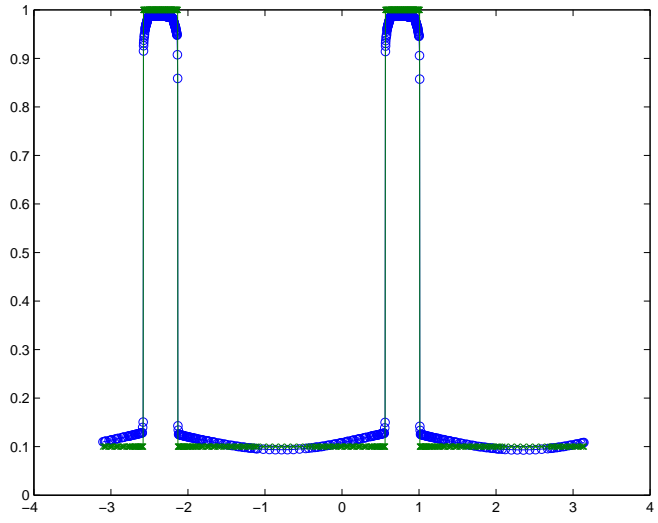

(a) $L^{2}$-norm error of $\gamma=5.9734 e-03, \beta=10^{-6}$

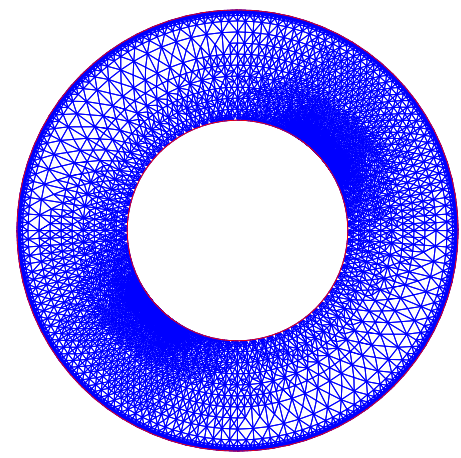

(b) Final mesh with 9401 nodes by adaptive Algorithm 3.1

Figure 4: Exact and numerically reconstructed Robin coefficients (left) and adaptively generated final mesh (right) in Example 2.

be highly discontinuous, which is expressed in the polar coordinate as

$$
\gamma(1, \theta)= \begin{cases}1 & \text { if } \sin (2 \theta)>0.9 \\ 0.1 & \text { otherwise }\end{cases}
$$

restricted on the inner boundary $\Gamma_{i}$. It behaves like two narrowly banded Delta functions; see Figure 4(a) (green). Figure 3(a) provides a graphical representation of the true solution $u$. An approximate Robin coefficient (blue) reconstructed by Algorithm 3.1, starting from the initial mesh in Figure 3(b), is depicted in Figure 4(a) and the corresponding adaptively generated final mesh in Figure 4(b). We observe that the reconstructed Robin coefficient has well detected the location and height of the strongly discontinuous exact coefficient, in view of the severe ill-posedness of the nonlinear inverse problem and the very poor constant initial guess of $\gamma=0.2$. Moreover, as one expects, the mesh refinements are correctly centered around two highly spiky regions to resolve the singularities of the strong discontinuities of the true Robin coefficient.

\section{Concluding remark}

We have proposed in this work an adaptive finite element method for recovering the Robin coefficient and established its convergence. With a general yet practical assumption imposed in the module MARK, discrete solutions produced by the adaptive algorithm are proved to converge to some exact Robin coefficient, state and costate variables.

One of the major difficulties in the analysis is a lack of convexity in the objective functional $\mathcal{J}(\gamma)$ for the Robin inverse problem, so we are unable to establish a reliable bound of the error in terms of an error estimator as in the existing theories for a posterior error estimates for direct partial differential equations or PDE-constrained optimal control problems. To overcome the difficulty, we have made use of some techniques in nonlinear optimization to study a limiting saddle-point system and the solution of this limiting problem is proved to satisfy the optimality conditions for the continuous problem. In our proofs a density argument is also utilized to handle a variational inequality. Then some computable quantities are derived only from our convergence analysis and serve as the effective estimator in our adaptive algorithm to guarantee the desired convergence. Numerical results show that the adaptive algorithm is efficient in the reconstruction of Robin coefficients. 
We think the approach in this work can be extended for the convergence analysis of AFEMs for other nonlinear inverse problems.

\section{Acknowledgements}

All the numerical experiments in this work are carried out by Mr. Man-chun Yuen, to whom the authors are truly grateful for his great efforts and professional help. Furthermore, the authors wish to thank two anonymous referees and the board member for many helpful and insightful comments and suggestions, which have improved the presentation and quality of the work greatly.

\section{References}

[1] M. Ainsworth and J. T. Oden, A Posteriori Error Estimation in Finite Element Analysis, Pure and Applied Mathematics, Wiley-Interscience, New York, 2000.

[2] W. Bangerth and A. Joshi, Adaptive finite element methods for the solution of inverse problems in optical tomography, Inverse Problems, 24 (2008), 1-22.

[3] R. Becker, H. Kapp and R. Rannacher, Adaptive finite element methods for optimal control of partial differential equations: Basic concept, SIAM J. Control Optim., 39 (2000), 113-132.

[4] R. Becker and B. Vexler, A posteriori error estimation for finite element discretization of parameter identification problems, Numer. Math., 96 (2004), 435-459.

[5] L. Beilina and M. V. Klibanov, A posteriori error estimates for the adaptivity technique for the Tikhonov functional and global convergence for a coefficient inverse problem, Inverse Problems, 26 (2010), 045012 (27pp).

[6] L. Belenki, L. Diening and C. Kreuzer, Optimality of an adaptive finite element method for the p-Laplacian equation, IMA J. Numer. Anal., 32 (2012), 484-510.

[7] P. Binev, W. Dahmen and R. DeVore, Adaptive finite element methods with convergence rates, Numer. Math., 97 (2004), 219-268.

[8] S Busenberg and W. Fang, Identification of semiconductor contact resistivity, Quar. J. Appl. Math., 49 (1991), 639-649.

[9] J. M. Cascon, C. Kreuzer, R. H. Nochetto and K. G. Siebert, Quasi-optimal convergence rate for an adaptive finite element method, SIAM J. Numer. Anal., 46 (2008), 2524-2550.

[10] S. Chaabane, C. Elhechmi and M. Jaoua, A stable recovery method for the Robin inverse problem, Math. Comput. Simul., 66 (2004), 367-383.

[11] S. Chaabane, I. Fellah, M. Jaoua and J. Leblond, Logarithmic stability estimates for a Robin coefficient in twodimensional Laplace inverse problems, Inverse Problems, 20 (2004), 47-59.

[12] S. Chaabane, J. Ferchichi and K. Kunisch, Differentiability properties of the $L^{1}$-tracking functional and application to the Robin inverse problem, Inverse Problems, 20 (2004), 1083-1097.

[13] S. Chaabane and M. Jaoua, Identification of Robin coefficients by the means of boundary measurements, Inverse Problems, 15 (1999), 1425-1438.

[14] S. Chantasiriwan, Inverse determination of steady-state heat transfer coefficient, Int. Comm. Heat Mass Transfer, 27 (2000), 1155-1164. 
[15] P. G. Ciarlet, Finite element methods for elliptic problems, North-Holland, Amsterdam, 1978.

[16] L. Diening and C. Kreuzer, Linear convergence of an adaptive finite element method for the p-Laplacian equation. SIAM J. Numer. Anal., 46 (2008), 614-638.

[17] L. E. Evans and R. F. Gariepy, Measure Theory and Fine Properties of Functions, CRC Press, Boca Raton, Florida, 1992.

[18] W. Fang and E. Cumberbatch, Inverse problems for metal oxide semiconductor field-effect transistor contact resistivity, SIAM J. Appl. Math., 52 (1992), 699-709.

[19] W. Fang and M. Lu, A fast collocation method for an inverse boundary value problem, Internat. J. Numer. Methods Engrg., 59 (2004), 1563-1585.

[20] D. Fasino and G. Inglese, An inverse Robin problem for Laplaces equation: theoretical results and numerical methods, Inverse Problems, 15 (1999), 41-48.

[21] T. Feng, N. Yan and W. Liu, Adaptive finite element methods for the identification of distributed parameters in elliptic equation, Adv. Comput. Math., 29 (2008), 27-53.

[22] E. M. Garau and P. Morin, Convergence and quasi-optimality of adaptive FEM for Steklov eigenvalue problems, IMA J. Num. Anal., 31 (2011), 914-946.

[23] E. M. Garau, P. Morin and C. Zuppa, Convergence of adaptive finite element methods for eigenvalue problems, Math. Models Methods Appl. Sci. 19 (2009), 721-747.

[24] E. M. Garau, P. Morin and C. Zuppa, Convergence of an adaptive Kačanov FEM for quasi-linear problems, Appl. Num. Math., 61 (2011), 512-529.

[25] E. M. Garau, P. Morin and C. Zuppa, Quasi-optimal convergence rate of an AFEM for quasilinear problems of monotone type, Numerical Mathematics: Theory, Methods and Applications 5 (2012), 131-156.

[26] A. Gaevskaya, R. Hoppe, Y. Iliash and M. Kieweg Convergence analysis of an adaptive finite element method for distributed control problems with control constraints, Proc. Conf. Optimal Control for PDEs, Oberwolfach, Germany (G. Leugering et al.; eds.), Birkhäuser, Basel, 2007.

[27] M. Hintermüller, R. Hoppe, Goal-oriented adaptivity in pointwise state constrained optimal control of partial differential equations, SIAM J. Control Optim., 48 (2010), 5468-5487.

[28] M. Hintermüller, R. Hoppe, Y. Iliash and M. Kieweg, An a posteriori error analysis of adaptive finite element methods for distributed elliptic control problems with control constraints, ESAIM, Control Optim. Calc. Var., 14 (2008), 540-560.

[29] G. Inglese, An inverse problem in corrosion detection, Inverse Problems, 13 (1997), 977-994.

[30] B. Jin and J. Zou, Numerical estimation of piecewise constant Robin coefficient, SIAM J. Control. Optim., 48 (2009), 1977-2002.

[31] B. Jin and J. Zou, Numerical estimation of the Robin coefficient in a stationary diffusion equation, IMA J. Numer. Anal., 30 (2010), 677-701.

[32] P. G. Kaup and F. Santosa, Nondestructive evaluation of corrosion damage using electrostatic measurements, J. Nondestr. Eval., 14 (1995), 127-136. 
[33] J. Li, J. Xie and J. Zou, An adaptive finite element reconstruction of distributed fluxes, Inverse Problems, 27 (2011), 075009 (25pp).

[34] R. Li, W. Liu, H. Ma and T. Tang, Adaptive finite element approximation for distributed elliptic optimal control problems SIAM J. Control Optim., 41 (2002), 1321-1349.

[35] F. Lin and W. Fang, A linear integral equation approach to the Robin inverse problem, Inverse Problems, 21 (2005), 1757-1772.

[36] W. Liu and N. Yan, A posteriori error estimates for distributed convex optimal control problems, Adv. Comput. Math., 15 (2001), 285-309.

[37] T. J. Martin and G. S. Dulikravich, Inverse determination of steady heat convection coefficient distribution, J. Heat Transfer, 120 (1998), 328-334.

[38] P. Morin, K. G. Siebert and A. Veeser, A basic convergence result for conforming adaptive finite elements, Math. Models Methods Appl. Sci., 18 (2008), 707-737.

[39] R. H. Nochetto, K. G. Siebert and A. Veeser, Theory of adaptive finite element methods: an introduction, Multiscale, Nonlinear and Adaptive Approximation (R. A. DeVore and A. Kunoth, Eds), Springer, New York, 2009, 409-542.

[40] L. R. Scott and S. Zhang, Finite element interpolation of nonsmooth functions satisfying boundary conditions, Math. Comp., 54 (1990), 483-493.

[41] K. G. Siebert, A convergence proof for adaptive finite elements without lower bounds, IMA J. Numer. Anal., 31 (2011), 947-970.

[42] R. Stevenson, Optimality of a standard adaptive finite element method, Found. Comput. Math., 7 (2007), 245-269.

[43] R. Stevenson, The completion of locally refined simplicial partitions created by bisection, Math. Comp., 77 (2008), 227-241.

[44] C. Traxler, An algorithm for adaptive mesh refinement in $n$ dimensions, Computing, 59 (1997), 115-137.

[45] R. Verfürth, A Review of A Posteriori Estimation and Adaptive Mesh-Refinement Techniques, Wiley-Teubner, Chichester, New York, Stuttgart, 1996.

[46] F. M. White, Heat and Mass Transfer, Addison-Wesley, Reading, MA, 1988.

[47] Y. Xu and J. Zou, Convergence of an adaptive finite element method for distributed flux reconstruction, to appear in Math. Comp. and preprint available at www.arXiv.org (arXiv: 1309.2101). 